\title{
AN OPTIMAL TRANSPORTATION METRIC FOR SOLUTIONS OF THE CAMASSA-HOLM EQUATION*
}

\author{
ALBERTO BRESSAN ${ }^{\dagger}$ AND MASSIMO FONTE $\ddagger$
}

Dedicated to Prof. Joel Smoller in the occasion of his 70-th birthday

\begin{abstract}
In this paper we construct a global, continuous flow of solutions to the Camassa-Holm equation on the entire space $H^{1}$. Our solutions are conservative, in the sense that the total energy $\int\left(u^{2}+u_{x}^{2}\right) d x$ remains a.e. constant in time. Our new approach is based on a distance functional $J(u, v)$, defined in terms of an optimal transportation problem, which satisfies $\frac{d}{d t} J(u(t), v(t)) \leq$ $\kappa \cdot J(u(t), v(t))$ for every couple of solutions. Using this new distance functional, we can construct arbitrary solutions as the uniform limit of multi-peakon solutions, and prove a general uniqueness result.
\end{abstract}

Key words. Camassa-Holm equation, optimal transportation metric, conservative solution

AMS subject classifications. 35G25, 35Q51, 49J20

1. Introduction. The Camassa-Holm equation can be written as a scalar conservation law with an additional integro-differential term:

$$
u_{t}+\left(u^{2} / 2\right)_{x}+P_{x}=0,
$$

where $P$ is defined as a convolution:

$$
P \doteq \frac{1}{2} e^{-|x|} *\left(u^{2}+\frac{u_{x}^{2}}{2}\right)
$$

For the physical motivations of this equation we refer to [CH], [CM1], [CM2], [J]. Earlier results on the existence and uniqueness of solutions can be found in [XZ1], [XZ2]. One can regard (1.1) as an evolution equation on a space of absolutely continuous functions with derivatives $u_{x} \in \mathbf{L}^{2}$. In the smooth case, differentiating (1.1) w.r.t. $x$ one obtains

$$
u_{x t}+u u_{x x}+u_{x}^{2}-\left(u^{2}+\frac{u_{x}^{2}}{2}\right)+P=0 .
$$

Multiplying (1.1) by $u$ and (1.3) by $u_{x}$ we obtain the two conservation laws with source term

$$
\begin{gathered}
\left(\frac{u^{2}}{2}\right)_{t}+\left(\frac{u^{3}}{3}+u P\right)_{x}=u_{x} P, \\
\left(\frac{u_{x}^{2}}{2}\right)_{t}+\left(\frac{u u_{x}^{2}}{2}-\frac{u^{3}}{3}\right)_{x}=-u_{x} P .
\end{gathered}
$$

As a consequence, for regular solutions the total energy

$$
E(t) \doteq \int\left[u^{2}(t, x)+u_{x}^{2}(t, x)\right] d x
$$

\footnotetext{
*Received June 7, 2005; accepted for publication November 5, 2005.

$\dagger$ Department of Mathematics, Penn State University, University Park, Pa. 16802 USA (bressan @math.psu.edu).

$\ddagger$ S.I.S.S.A., Via Beirut 4, Trieste 34014, Italy (fonte@sissa.it).
} 
remains constant in time.

As in the case of scalar conservation laws, by the strong nonlinearity of the equations, solutions with smooth initial data can lose regularity in finite time. For the Camassa-Holm equation (1.1), however, the uniform bound on $\left\|u_{x}\right\|_{\mathbf{L}^{2}}$ guarantees that only the $\mathbf{L}^{\infty}$ norm of the gradient can blow up, while the solution $u$ itself remains Hölder continuous at all times.

In order to construct global in time solutions, two main approaches have recently been introduced. On one hand, one can add a small diffusion term in the right hand side of (1.1), and recover solutions of the original equations as a vanishing viscosity limit [CHK1, CHK2]. An alternative technique, developed in [BC2], relies on a new set of independent and dependent variables, specifically designed with the aim of "resolving" all singularities. In terms of these new variables, the solution to the Cauchy problem becomes regular for all times, and can be obtained as the unique fixed point of a contractive transformation.

In the present paper, we implement yet another approach to the Camassa-Holm equation. As a starting point we consider all multi-peakon solutions, of the form

$$
u(t, x)=\sum_{i=1}^{N} p_{i}(t) e^{-\left|x-q_{i}(t)\right|} .
$$

These are obtained by solving the system of O.D.E's

$$
\left\{\begin{array}{l}
\dot{q}_{i}=\sum_{j} p_{j} e^{-\left|q_{i}-q_{j}\right|} \\
\dot{p}_{i}=\sum_{j \neq i} p_{i} p_{j} \operatorname{sign}\left(q_{i}-q_{j}\right) e^{-\left|q_{i}-q_{j}\right|} .
\end{array}\right.
$$

It is well known that this can be written in hamiltonian form:

$$
\left\{\begin{array}{l}
\dot{q}_{i}=\frac{\partial}{\partial p_{i}} H(p, q), \\
\dot{p}_{i}=-\frac{\partial}{\partial q_{i}} H(p, q),
\end{array} \quad H(p, q) \doteq \frac{1}{2} \sum_{i, j} p_{i} p_{j} e^{-\left|q_{i}-q_{j}\right|} .\right.
$$

If all the coefficients $p_{i}$ are initially positive, then they remain positive and bounded for all times. The solution $u=u(t, x)$ is thus uniformly Lipschitz continuous. We stress, however, that here we are not making any assumption about the signs of the $p_{i}$. In a typical situation, two peakons can cross at a finite time $\tau$. As $t \rightarrow \tau-$ their strengths $p_{i}, p_{j}$ and positions $q_{i}, q_{j}$ will satisfy

$$
\begin{gathered}
p_{i}(t) \rightarrow+\infty, \quad p_{j}(t) \rightarrow-\infty, \quad p_{i}(t)+p_{j}(t) \rightarrow \bar{p}, \\
q_{i}(t) \rightarrow \bar{q}, \quad q_{j}(t) \rightarrow \bar{q}, \quad q_{i}(t)<q_{j}(t) \quad \text { for } t<\tau,
\end{gathered}
$$

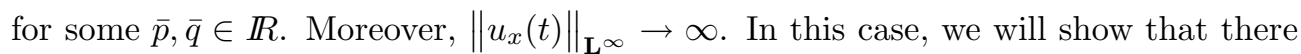
exists a unique way to extend the multi-peakon solution beyond the interaction time, so that the total energy is conserved.

Having constructed a set of "multi-peakon solutions", our main goal is to show that these solutions form a continuous semigroup, whose domain is dense in the space 
$H^{1}(\mathbb{R})$. Taking the unique continuous extension, we thus obtain a continuous semigroup of solutions of (1.1), defined on the entire space $H^{1}$.

The particular metric, used to derive the Lipschitz continuity of the semigroup, is the most novel feature of our paper. Indeed, one easily checks that the flow map $\Phi_{t}: u(0) \mapsto u(t)$ cannot be continuous as a map from $H^{1}$ into itself, or from $\mathbf{L}^{2}$ into itself. Distances defined in terms of convex norms perform well in connection with linear problems, but occasionally fail when nonlinear features become dominant. In the present setting, we construct a new distance $J(u, v)$ between functions $u, v \in H^{1}$, defined by a problem of optimal transportation. Roughly speaking, $J(u, v)$ will be the minimum cost in order to transport the mass distribution with density $1+u_{x}^{2}$ located on the graph of $u$ onto the mass distribution with density $1+v_{x}^{2}$ located on the graph of $v$. See Section 3 for details. With this definition of distance, our main result shows that

$$
\left|\frac{d}{d t} J(u(t), v(t))\right| \leq C \cdot J(u(t), v(t))
$$

for some constant $C$ and any couple of multi-peakon solutions $u, v$. Moreover, $J\left(u_{n}, u\right) \rightarrow$ 0 implies the uniform convergence $\left\|u_{n}-u\right\|_{\mathbf{L}^{\infty}} \rightarrow 0$. The distance functional $J$ thus provides the ideal tool to measure continuous dependence on the initial data for solutions to the Camassa-Holm equation. Earlier applications of distances defined in terms of optimal transportation problems can be found in the monograph [V].

The issue of uniqueness of solutions must here be discussed in greater detail. For a multi-peakon solution, as long as all coefficients $p_{i}$ remain bounded, the solution to the system of ODE's (1.8) is clearly unique. For each time $t$, call $\mu_{t}$ the measure having density $u^{2}(t)+u_{x}^{2}(t)$ w.r.t. Lebesgue measure. Consider a time $\tau$ where a positive and a negative peakon collide, according to (1.9)-(1.10). As $t \rightarrow \tau-$, we have the weak convergence $\mu_{t} \rightarrow \mu_{\tau}$ for some positive measure $\mu_{\tau}$ which typically contains a Dirac mass at the point $\bar{q}$. By energy conservation, we thus have

$$
\int\left[u^{2}(\tau, x)+u_{x}^{2}(\tau, x)\right] d x+\mu_{\tau}(\{\bar{q}\})=\lim _{t \rightarrow \tau-} \int\left[u^{2}(\tau, x)+u_{x}^{2}(\tau, x)\right] d x=E(\tau-) .
$$

There are now two natural ways to prolong the multi-peakon solution beyond time $\tau$ : a conservative solution, such that

$$
E(t)=\int\left[u^{2}(t, x)+u_{x}^{2}(t, x)\right] d x=E(\tau-) \quad t>\tau,
$$

or a dissipative solution, where all the energy concentrated at the point $\bar{q}$ is lost. In this case

$$
E(t)=\int\left[u^{2}(t, x)+u_{x}^{2}(t, x)\right] d x=E(\tau-)-\mu_{\tau}(\{\bar{q}\}) \quad t>\tau .
$$

For $t>\tau$, the dissipative solution is obtained by simply replacing the two peakons $p_{i}, p_{j}$ with one single peakon of strength $\bar{p}$, located at $x=\bar{q}$. On the other hand, as we will show in Section 2, the conservative solution contains two peakons emerging from the point $\bar{q}$. As $t \rightarrow \tau+$, their strengths and positions satisfy again (1.9), while (1.10) is replaced by

$$
q_{i}(t) \rightarrow \bar{q}, \quad q_{j}(t) \rightarrow \bar{q}, \quad q_{i}(t)>q_{j}(t) \text { for } t>\tau
$$


The vanishing viscosity approach in [CHK1, CHK2] singles out the dissipative solutions. These can also be characterized by the Oleinik type estimate

$$
u_{x}(t, x) \leq C\left(1+t^{-1}\right),
$$

valid for $t>0$ at a.e. $x \in \mathbb{R}$. On the other hand, the coordinate transformation approach in [BC2] and the present one, based on optimal transport metrics, appear to be well suited for the study of both conservative and dissipative solutions.

In the present paper we focus on conservative solutions to the $\mathrm{C}-\mathrm{H}$ equation in the spatially periodic case. The assumption of spatial periodicity allows us to concentrate on the heart of the matter, i.e. the uniqueness and stability of solutions beyond the time of singularity formation. It will spare us some technicalities, such as the analysis of the tail decay of $u, u_{x}$ as $x \rightarrow \pm \infty$. The construction of an optimal transportation metric in connection with dissipative solutions to the $\mathrm{C}-\mathrm{H}$ equation is significantly different, and will be carried out in a forthcoming paper. The main ingredients can already be found in the paper [BC1], devoted to dissipative solutions of the HunterSaxton equation.

As initial data, we take

$$
u(0, x)=\bar{u}(x),
$$

with $\bar{u}$ in the space $H_{\mathrm{p} e r}^{1}$ of of periodic, absolutely continuous functions $u$ with derivative $u_{x} \in \mathbf{L}_{\text {per }}^{2}$. To fix the ideas, we assume that the period is 1 , so that

$$
u(x+1)=u(x) \quad x \in \mathbb{R} .
$$

On $H_{\mathrm{per}}^{1}$ we shall use the norm

$$
\|u\|_{H_{\mathrm{per}}^{1}} \doteq\left(\int_{0}^{1}|u(x)|^{2} d x+\int_{0}^{1}\left|u_{x}(x)\right|^{2} d x\right)^{1 / 2} .
$$

Our main results can be stated as follows.

Theorem 1. For each initial data $\bar{u} \in H_{\mathrm{per}}^{1}$, there exists a solution $u(\cdot)$ of the Cauchy problem (1.1), (1.13). Namely, the map $t \mapsto u(t)$ is Lipschitz continuous from $\mathbb{R}$ into $\mathbf{L}_{\mathrm{per}}^{2}$, satisfies (1.13) at time $t=0$, and the identity

$$
\frac{d}{d t} u=-u u_{x}-P_{x}
$$

is satisfied as an equality between elements in $\mathbf{L}_{\mathrm{per}}^{2}$ at a.e. time $t \in \mathbb{R}$. This same map $t \mapsto u(t)$ is continuously differentiable from $\mathbb{R}$ into $\mathbf{L}_{\mathrm{per}}^{p}$ and satisfies (1.14) at a.e. time $t \in \mathbb{R}$, for all $p \in[1,2[$. The above solution is conservative in the sense that, for a.e. $t \in \mathbb{R}$,

$$
E(t)=\int_{0}^{1}\left[u^{2}(t, x)+u_{x}^{2}(t, x)\right] d x=E^{\bar{u}} \doteq \int_{0}^{1}\left[\bar{u}^{2}(x)+\bar{u}_{x}^{2}(x)\right] d x .
$$

TheOREM 2. Conservative solutions to (1.1) can be constructed so that they constitute a continuous flow $\Phi$. Namely, there exists a distance functional $J$ on $H_{\mathrm{per}}^{1}$ such that

$$
\frac{1}{C} \cdot\|u-v\|_{\mathbf{L}_{\mathrm{per}}^{1}} \leq J(u, v) \leq C \cdot\|u-v\|_{H_{\mathrm{per}}^{1}}
$$


for all $u, v \in H_{\mathrm{per}}^{1}$ and some constant $C$ uniformly valid on bounded sets of $H_{\mathrm{per}}^{1}$. Moreover, for any two solutions $u(t)=\Phi_{t} \bar{u}, v(t)=\Phi_{t} \bar{v}$ of (1.1), the map $t \mapsto$ $J(u(t), v(t))$ satisfies

$$
\begin{gathered}
J(u(t), \bar{u}) \leq C_{1} \cdot|t|, \\
J(u(t), v(t)) \leq J(\bar{u}, \bar{v}) \cdot e^{C_{2}|t|}
\end{gathered}
$$

for a.e. $t \in \mathbb{R}$ and constants $C_{1}, C_{2}$, uniformly valid as $u, v$ range on bounded sets of $H_{p e r}^{1}$.

Somewhat surprisingly, all the properties stated in Theorem 1 are still not strong enough to single out a unique solution. To achieve uniqueness, an additional condition is needed.

THEOREM 3. Conservative solutions $t \mapsto u(t)$ of (1.1) can be constructed with the following additional property:

For each $t \in \mathbb{R}$, call $\mu_{t}$ the absolutely continuous measure having density $u^{2}+u_{x}^{2}$ w.r.t. Lebesgue measure. Then, by possibly redefining $\mu_{t}$ on a set of times of measure zero, the map $t \mapsto \mu_{t}$ is continuous w.r.t. the topology of weak convergence of measures. It provides a measure-valued solution to the conservation law

$$
w_{t}+(u w)_{x}=\left(u^{3}-2 u P^{u}\right)_{x}
$$

The solution of the Cauchy problem (1.1), (1.13) satisfying the properties stated in Theorem 1 and this additional condition is unique.

The plan of the paper is as follows. In Section 2 we derive some elementary properties of multi-peakon solutions and show that any initial data can be approximated in $H_{\text {per }}^{1}$ by a finite sum of peakons. In Section 3 we introduce our distance functional $J(u, v)$ and study its relations with other distances defined by Sobolev norms. The continuity of the flow (1.1), together with the key estimates (1.17)-(1.18) are then proved in the following two sections. The proofs of Theorems 1 and 2 are completed in Section 6. The uniqueness result stated in Theorem 3 is proved in Section 7. As a corollary, we also show that in a multi-peakon solution the only possible interactions involve exactly two peakons: one positive and one negative. In particular, no triple interactions can ever occur.

2. Multipeakon solutions. By a periodic peakon we mean a function of the form

$$
u(x)=p \chi(x-q), \quad \chi(x) \doteq \sum_{n \in \mathbb{Z}} e^{-|x-n|} .
$$

Observe that the periodic function $\chi$ satisfies

$$
\begin{array}{ll}
\chi(-x)=\chi(x)=\chi(x+1) & x \in \mathbb{R}, \\
\chi(x)=\frac{e^{x}+e^{1-x}}{e-1} & x \in[0,1] .
\end{array}
$$

We begin this section by observing that any periodic initial data can be approximated by a periodic multi-peakon. 
Lemma 1. Let $f \in H_{\mathrm{per}}^{1}$. Then for any $\varepsilon>0$ there exists periodic multi-peakon $g$, of the form

$$
g(x)=\sum_{i=1}^{N} p_{i} \sum_{n \in \mathbb{Z}} e^{-\left|x-q_{i}-n\right|}=\sum_{i=1}^{N} p_{i} \chi\left(x-q_{i}\right)
$$

such that

$$
\|f-g\|_{H_{\mathrm{per}}^{1}}<\varepsilon
$$

Proof. By taking a suitable mollification, we can approximate $f$ with a periodic function $\tilde{f} \in \mathcal{C}^{\infty}$, so that

$$
\|f-\tilde{f}\|_{H_{\mathrm{per}}^{1}}<\varepsilon / 2
$$

Next, we observe that

$$
\frac{1}{2}\left(e^{-|x|}-\frac{\partial^{2}}{\partial x^{2}} e^{-|x|}\right)=\delta_{0}
$$

where $\delta_{0}$ denotes the Dirac distribution concentrating a unit mass at the origin. We can thus write $\tilde{f}$ as a convolution:

$$
\begin{gathered}
\tilde{f}=\delta_{0} * \tilde{f}=\frac{1}{2}\left(e^{-|x|}-\frac{\partial^{2}}{\partial x^{2}} e^{-|x|}\right) * \tilde{f}=e^{-|x|} *\left(\frac{\tilde{f}-\tilde{f}^{\prime \prime}}{2}\right), \\
\tilde{f}(x)=\int_{0}^{1} \chi(x-y) \cdot \frac{\tilde{f}(y)-\tilde{f}^{\prime \prime}(y)}{2} d y .
\end{gathered}
$$

The above integral can now be approximated with a Riemann sum

$$
g(x)=\sum_{i=1}^{N} p_{i} \chi\left(x-q_{i}\right), \quad \quad p_{i}=\int_{(i-1) / N}^{i / N} \frac{\tilde{f}(y)-\tilde{f}^{\prime \prime}(y)}{2} d y .
$$

Choosing $N$ sufficiently large we obtain $\|\tilde{f}-g\|_{H_{p e r}^{1}}<\varepsilon / 2$. Together with (2.4) this yields the result.

Next, we show how to construct a unique conservative solution, for multi-peakon initial data. As long as the locations $q_{i}$ of the peakons remain distinct, this can be obtained by solving the Hamiltonian system of O.D.E's (1.8). However, at a time $\tau$ where two or more peakons interact, the corresponding strengths $p_{i}$ become unbounded. A suitable transformation of variables is needed, in order to resolve the singularity and uniquely extend the solution beyond the interaction time.

Lemma 2. Let $\bar{u}$ be any periodic, multi-peakon initial data. Then the Cauchy problem (1.1), (1.13) has a global, conservative multi-peakon solution defined for all $t \in \mathbb{R}$. The set $\mathcal{I}$ of times where two or more peakons interact is at most countable. Moreover, for all $t \notin \mathcal{I}$, the energy conservation (1.15) holds.

Proof. The solution can be uniquely constructed by solving the hamiltonian system (1.8), up to the first time $\tau$ where two or more peakons interact. We now 
show that there exists a unique way to prolong the solution for $t>\tau$, in terms of two outgoing peakons. To fix the ideas, call

$$
\bar{q}=\lim _{t \rightarrow \tau-} q_{i}(t) \quad i=1, \ldots, k
$$

the place where the interaction occurs, and let $p_{1}(t), \ldots, p_{k}(t)$ be the strengths of the interacting peakons. Later in this paper we will show that only the case $k=2$ can actually occur, but at this stage we need to consider the more general case. We observe that the strengths $p_{k+1}, \ldots, p_{N}$ of the peakons not involved in the interaction remain continuous at time $\tau$. Moreover, by (1.8) there exists the limit

$$
\bar{p}=\lim _{t \rightarrow \tau-} \sum_{i=1}^{k} p_{i}(t)
$$

We can thus write

$$
u(\tau, x)=\lim _{t \rightarrow \tau-} \sum_{i=1}^{N} p_{i}(t) e^{-\left|x-q_{i}(t)\right|}=\bar{p} e^{-|x-\bar{q}|}+\sum_{i=k+1}^{N} p_{i}(\tau) e^{-\left|x-q_{i}(\tau)\right|} .
$$

For $t>\tau$, we shall prolong the solution with two peakons emerging from the point $\bar{q}$. The strength of these two peakons will be uniquely determined by the requirement of energy conservation (1.15).

Call $\xi^{-}(t), \xi^{+}(t)$ respectively the position of the smallest and largest characteristic curves passing through the point $(\tau, \bar{q})$, namely

$$
\begin{array}{ll}
\xi^{-}(t) \doteq \min \{\xi(t) ; \quad \xi(\tau)=\bar{q}, \quad \dot{\xi}(s)=u(s, \xi(s)) & \text { for all } s \in[\tau-h, \tau+h]\} \\
\xi^{+}(t) \doteq \max \{\xi(t) ; \quad \xi(\tau)=\bar{q}, \quad \dot{\xi}(s)=u(s, \xi(s)) & \text { for all } s \in[\tau-h, \tau+h]\} .
\end{array}
$$

Moreover, define

$$
e_{(\tau, \bar{q})} \doteq \lim _{t \rightarrow \tau-} \int_{\xi^{-}(t)}^{\xi^{+}(t)} u_{x}^{2}(t, x) d x
$$

The existence of this limit follows from the balance law (1.5). This describes how much energy is concentrated at the interaction point.

For $t>\tau$ the solution will contain the peakons $p_{k+1}, \ldots, p_{N}$, located at $q_{k+1}, \ldots, q_{N}$, together with the two outgoing peakons $p_{1}, p_{2}$, located at $q_{1}<q_{2}$. The behavior of $p_{i}, q_{i}$ for $i \in\{k+1, \ldots, N\}$ is still described by a system of O.D.E's as in (1.8). However, to describe the evolution of $p_{1}, p_{2}, q_{1}, q_{2}$ one has to use a different set of variables, resolving the singularity occurring at $(\tau, \bar{q})$. As $t \rightarrow \tau+$ we expect (1.9), (1.12) to hold. To devise a suitable set of rescaled variables, we observe that, by (1.3),

$$
\frac{d}{d t} u_{x}(t, \xi(t))=-\frac{1}{2} u_{x}^{2}(t, \xi(t))+\left[u^{2}-P\right]
$$

along any characteristic curve $t \mapsto \xi(t)$ emerging from the point $\bar{q}$. Since $u, P$ remain uniformly bounded, one has

$$
u_{x}(t, x) \approx \frac{2}{t-\tau} \quad t>\tau, \quad x \in\left[q_{1}(t), q_{2}(t)\right] .
$$


The total amount of energy concentrated in the interval between the two peakons is given by

$$
\begin{aligned}
\int_{q_{1}(t)}^{q_{2}(t)}\left[u^{2}(t, x)+u_{x}^{2}(t, x)\right] d x & \approx\left(\frac{u\left(q_{2}\right)-u\left(q_{1}\right)}{q_{2}-q_{1}}\right)^{2} \cdot\left(q_{2}-q_{1}\right) \\
& \approx \frac{\left[\left(p_{2}-p_{1}\right)\left(1-e^{-\left|q_{2}-q_{1}\right|}\right)\right]^{2}}{q_{2}-q_{1}} \\
& \approx\left(p_{2}-p_{1}\right)^{2}\left(q_{2}-q_{1}\right) \approx e_{(\tau, \bar{q})} .
\end{aligned}
$$

The previous heuristic analysis suggests that, in order to resolve the singularities, we should work with the variables

$$
z=p_{1}+p_{2}, \quad w=2 \arctan \left(p_{2}-p_{1}\right), \quad \eta=q_{2}+q_{1}, \quad \zeta=\left(p_{2}-p_{1}\right)^{2}\left(q_{2}-q_{1}\right),
$$

together with $p_{k+1}, \ldots, p_{N}, \quad q_{k+1}, \cdots, q_{N}$. To simplify the following calculations we here assume $0<q_{1}<q_{2}<q_{k+1}<\ldots<q_{N}<1$, which is not restrictive. Let $\chi$ be as in (2.1) and define $\tilde{\chi}$ as the (discontinuous) 1-periodic function such that $\tilde{\chi}(x) \doteq \frac{e^{1-x}-e^{x}}{e-1}$ for $0<x<1$. In terms of the new variables, the original system of equations (1.8) can be rewritten as

$$
\begin{aligned}
& \dot{z}=\cosh \left(\frac{\zeta}{2 \tan ^{2} \frac{w}{2}}\right) z \cos ^{2} \frac{w}{2} \sum_{j=k+1}^{N} p_{j} \chi\left(q_{j}-\frac{\eta}{2}\right)-\frac{\sinh \left(\frac{\zeta}{2 \tan ^{2} \frac{w}{2}}\right)}{\frac{1}{\tan \frac{w}{2}}} \sum_{j=k+1}^{N} p_{j} \tilde{\chi}\left(q_{j}-\frac{\eta}{2}\right) \\
& \dot{w}=\left(z^{2} \cos ^{2} \frac{w}{2}-\sin ^{2} \frac{w}{2}\right) \chi\left(\frac{\zeta}{\tan ^{2} \frac{w}{2}}\right)+2 \cosh \left(\frac{\zeta}{2 \tan ^{2} \frac{w}{2}}\right) z \cdot \sum_{j=k+1}^{N} p_{j} \chi\left(q_{j}-\frac{\eta}{2}\right) \\
& +2 \sinh \left(\frac{\zeta}{2 \tan ^{2} \frac{w}{2}}\right) \sin w \sum_{j=k+1}^{N} p_{j} \tilde{\chi}\left(q_{j}-\frac{\eta}{2}\right) \\
& \dot{\eta}=z\left[\chi(0)+\chi\left(\frac{\zeta}{\tan ^{2} \frac{w}{2}}\right)\right]+\cosh \left(\frac{\zeta}{2 \tan ^{2} \frac{w}{2}}\right) \sum_{j=k+1}^{N} p_{j} \chi\left(q_{j}-\frac{\eta}{2}\right) \\
& \dot{\zeta}=\frac{\chi(0)-\chi\left(\frac{\zeta}{\tan ^{2} \frac{w}{2}}\right)}{\frac{1}{\tan ^{2} \frac{w}{2}}} \zeta+\chi\left(\frac{\zeta}{\tan ^{2} \frac{w}{2}}\right) \frac{z^{2} \zeta}{\tan \frac{w}{2}}-\frac{\sinh \left(\frac{\zeta}{2 \tan ^{2} \frac{w}{2}}\right)}{\frac{1}{\tan ^{2} \frac{w}{2}}} \sum_{j=k+1}^{N} p_{j} \tilde{\chi}\left(q_{j}-\frac{\eta}{2}\right) \\
& +2 \frac{\zeta}{\tan \frac{w}{2}}\left[\cosh \left(\frac{\zeta}{2 \tan ^{2} \frac{w}{2}}\right) \frac{z}{\tan \frac{w}{2}} \sum_{j=k+1}^{N} p_{j} \chi\left(q_{j}-\frac{\eta}{2}\right)-\sinh \left(\frac{\zeta}{2 \tan ^{2} \frac{w}{2}}\right) \sum_{j=k+1}^{N} p_{j} \tilde{\chi}\left(q_{j}-\frac{\eta}{2}\right)\right] \\
& \dot{p}_{i}=p_{i}\left[\cosh \left(\frac{\zeta}{2 \tan ^{2} \frac{w}{2}}\right) z \cdot \chi\left(q_{i}-\frac{\eta}{2}\right)+\sinh \left(\frac{\zeta}{2 \tan ^{2} \frac{w}{2}}\right) \tan \frac{w}{2} \cdot \tilde{\chi}\left(q_{i}-\frac{\eta}{2}\right)\right] \\
& +p_{i} \sum_{j=k+1}^{N} p_{j} \operatorname{sign}\left(q_{i}-q_{j}\right) \chi\left(q_{i}-q_{j}\right) \\
& \dot{q}_{i}=\cosh \left(\frac{\zeta}{2 \tan ^{2} \frac{w}{2}}\right) z \cdot \chi\left(q_{i}-\frac{\eta}{2}\right)+\sinh \left(\frac{\zeta}{2 \tan ^{2} \frac{w}{2}}\right) \tan \frac{w}{2} \tilde{\chi}\left(q_{i}-\frac{\eta}{2}\right)+\sum_{j=k+1}^{N} p_{j} \chi\left(q_{i}-q_{j}\right)
\end{aligned}
$$

with initial data

$$
z(\tau)=\bar{p}, \quad w(\tau)=\pi, \quad \eta(\tau)=2 \bar{q}, \quad \zeta(\tau)=e_{(\tau, \bar{q})},
$$




$$
p_{i}(\tau)=\lim _{t \rightarrow \tau-} p_{i}(t), \quad q_{i}(\tau)=\lim _{t \rightarrow \tau-} q_{i}(t) \quad i=k+1, \ldots, N .
$$

For the above system of O.D.E's, a direct inspection reveals that the right hand side can be extended by continuity also at the value $w=\pi$, because all singularities are removable. This continuous extension is actually smooth, in a neighborhood of the initial data. Therefore, our Cauchy problem has a unique local solution. This provides a multi-peakon solution defined on some interval of the form $\left[\tau, \tau^{\prime}[\right.$, up to the next interaction time.

The case where two or more groups of peakons interact exactly at the same time $\tau$, but at different locations within the interval $[0,1]$, can be treated in exactly the same way. Since the total number of peakons (on a unit interval in the $x$-variable) does not increase, it is clear that the number of interaction times is at most countable. The solution can thus be extended to all times $t>0$, conserving its total energy.

3. A distance functional. In this section we shall construct a functional $J(u, v)$ which controls the distance between two solutions of the equation (1.1). All functions and measures on $\mathbb{R}$ are assumed to be periodic with period 1 . Let $\mathbf{T}$ be the unit circle, so that $\mathbf{T}=[0,2 \pi]$ with the endpoints 0 and $2 \pi$ identified. The distance $\left|\theta-\theta^{\prime}\right|_{*}$ between two points $\theta, \theta^{\prime} \in \mathbf{T}$ is defined as the smaller between the lengths of the two $\operatorname{arcs}$ connecting $\theta$ with $\theta^{\prime}$ (one clockwise, the other counterclockwise). We now consider the product space

$$
X \doteq \mathbb{R} \times \mathbb{R} \times \mathbf{T}
$$

with distance

$$
d \diamond((x, u, w), \quad(\tilde{x}, \tilde{u}, \tilde{w})) \doteq\left(|x-\tilde{x}|+|u-\tilde{u}|+|w-\tilde{w}|_{*}\right) \wedge 1
$$

where $a \wedge b \doteq \min \{a, b\}$. Let $\mathcal{M}(X)$ be the space of all Radon measures on $X$ which are 1-periodic w.r.t. the $x$-variable. To each 1-periodic function $u \in H_{\mathrm{per}}^{1}$ we now associate the positive measure $\sigma^{u} \in \mathcal{M}(X)$ defined as

$$
\sigma^{u}(A) \doteq \int_{\left\{x \in \mathbb{R}:\left(x, u(x), 2 \arctan u_{x}(x)\right) \in A\right\}}\left(1+u_{x}^{2}(x)\right) d x
$$

for every Borel set $A \subseteq \mathbb{R}^{2} \times \mathbf{T}$. Notice that the total mass of $\sigma^{u}$ over one period is

$$
\sigma^{u}([0,1] \times \mathbb{R} \times \mathbf{T})=1+\int_{0}^{1} u_{x}^{2}(x) d x .
$$

On this family of positive, 1-periodic Radon measures, we now introduce a kind of Kantorovich distance, related to an optimal transportation problem. Given the two measures $\sigma^{u}$ and $\sigma^{\tilde{u}}$, their distance $J(u, \tilde{u})$ is defined as follows.

Call $\mathcal{F}$ the family of all strictly increasing absolutely continuous maps $\psi: \mathbb{R} \mapsto \mathbb{R}$ which have an absolutely continuous inverse and satisfy

$$
\psi(x+n)=n+\psi(x) \quad \text { for every } n \in \mathbb{Z} .
$$


For a given $\psi \in \mathcal{F}$, we define the 1 -periodic, measurable functions $\phi_{1}, \phi_{2}: \mathbb{R} \mapsto[0,1]$ by setting

$$
\begin{aligned}
\phi_{1}(x) & \doteq \sup \left\{\theta \in[0,1] ; \quad \theta \cdot\left(1+u_{x}^{2}(x)\right) \leq\left(1+\tilde{u}_{x}^{2}(\psi(x))\right) \psi^{\prime}(x)\right\}, \\
\phi_{2}(\psi(x)) & \doteq \sup \left\{\theta \in[0,1] ; 1+u_{x}^{2}(x) \geq \theta \cdot\left(1+\tilde{u}_{x}^{2}(\psi(x))\right) \psi^{\prime}(x)\right\} .
\end{aligned}
$$

Observe that the above definitions imply $\max \left\{\phi_{1}(x), \phi_{2}(\psi(x))\right\}=1$ together with

$$
\phi_{1}(x)\left(1+u_{x}^{2}(x)\right)=\phi_{2}(\psi(x))\left(1+\tilde{u}_{x}^{2}(\psi(x))\right) \psi^{\prime}(x)
$$

for a.e. $x \in \mathbb{R}$. We now define

$$
\begin{aligned}
J^{\psi}(u, \tilde{u}) \doteq & \int_{0}^{1} d \diamond\left(\left(x, u(x), 2 \arctan u_{x}(x)\right),\left(\psi(x), \tilde{u}(\psi(x)), 2 \arctan \tilde{u}_{x}(\psi(x))\right)\right. \\
\cdot \phi_{1}(x)\left(1+u_{x}^{2}(x)\right) d x & \\
& +\int_{0}^{1}\left|\left(1+u_{x}^{2}(x)\right)-\left(1+\tilde{u}_{x}^{2}(\psi(x))\right) \psi^{\prime}(x)\right| d x .
\end{aligned}
$$

Of course, the integral is always computed over one period. Observe that $x \mapsto \psi(x)$ can be regarded as a transportation plan, in order to transport the measure $\sigma^{u}$ onto the measure $\sigma^{\tilde{u}}$. Since these two positive measures need not have the same total mass, we allow the presence of some excess mass, not transferred from one place to the other. The penalty for this excess mass is given by the second integral in (3.6). The factor $\phi_{1} \leq 1$ in the first integral indicates the percentage of the mass which is actually transported. Integrating (3.5) over one period, we find

$$
\int_{0}^{1} \phi_{1}(x)\left(1+u_{x}^{2}(x)\right) d x=\int_{0}^{1} \phi_{2}(y)\left(1+\tilde{u}_{x}^{2}(y)\right) d y .
$$

We can thus transport the measure $\phi_{1} \sigma^{u}$ onto $\phi_{2} \sigma^{\tilde{u}}$ by a map $\Psi:(x, u(x) \arctan$ $\left.u_{x}(x)\right) \mapsto\left(y, \tilde{u}(y), \arctan \tilde{u}_{x}(y)\right)$, with $y=\psi(x)$. The associated cost is given by the first integral in (3.6). Notice that in this case the measure $\phi_{2} \sigma^{\tilde{u}}$ is obtained as the push-forward of the measure $\phi_{1} \sigma^{u}$. We recall that the push-forward of a measure $\sigma$ by a mapping $\Psi$ is defined as $(\Psi \# \sigma)(A) \doteq \sigma\left(\Psi^{-1}(A)\right)$ for every measurable set $A$. Here $\Psi^{-1}(A) \doteq\{z ; \Psi(z) \in A\}$.

Our distance functional $J$ is now obtained by optimizing over all transportation plans, namely

$$
J(u, \tilde{u}) \doteq \inf _{\psi \in \mathcal{F}} J^{\psi}(u, \tilde{u})
$$

To check that (3.7) actually defines a distance, let $u, v, w \in H^{1}(\mathbb{R})$ be given.

1. Choosing $\psi(x)=x$, so that $\phi_{1}(x)=\phi_{2}(x)=1$, we immediately see that $J(u, u)=$ 0 . Moreover, if $J(u, \tilde{u})=0$, then by the definition of $d \diamond$ we have $\tilde{u}=u$.

2. Given $\psi \in \mathcal{F}$, define $\tilde{\psi}=\psi^{-1}$, so that $\tilde{\phi}_{1}=\phi_{2}, \tilde{\phi}_{2}=\phi_{1}$. This yields

$$
J \tilde{\psi}(\tilde{u}, u)=J \psi(u, \tilde{u}) .
$$


Hence $J(\tilde{u}, u)=J(u, \tilde{u})$.

3. Finally, to prove the triangle inequality, let $\psi^{b}, \psi^{\sharp}: \mathbb{R} \mapsto \mathbb{R}$ be two increasing diffeomorphisms satisfying (3.3), and let $\phi_{1}^{\mathrm{b}}, \phi_{1}^{\sharp}, \phi_{2}^{\mathrm{b}}, \phi_{2}^{\sharp}: \mathbb{R} \mapsto[0,1]$ be the corresponding functions, defined as in (3.4). We now consider the composition $\psi \doteq \psi^{\sharp} \circ \psi^{\mathrm{b}}$ and define the functions $\phi_{1}, \phi_{2}$ according to (3.4). Observing that

$$
\begin{gathered}
\phi_{1}(x) \geq \phi_{1}^{b}(x) \cdot \phi_{1}^{\sharp}\left(\psi^{b}(x)\right), \\
\phi_{2}(\psi(x))=\phi_{2}\left(\psi^{\sharp}\left(\psi^{b}(x)\right)\right) \geq \phi_{2}^{b}\left(\psi^{b}(x)\right) \cdot \phi_{2}^{\sharp}\left(\psi^{\sharp}\left(\psi^{b}(x)\right)\right),
\end{gathered}
$$

and recalling that the distance $d \diamond$ at (3.1) is always $\leq 1$, we conclude

$$
J \psi(u, w) \leq J^{\psi^{b}}(u, v)+J \psi^{\sharp}(v, w) .
$$

This implies the triangle inequality $J(u, v)+J(v, w) \geq J(u, w)$.

In the remainder of this section we study the relations between our distance functional $J$ and the distances determined by various norms.

Lemma 3. For any $u, v \in H_{\mathrm{per}}^{1}$ one has

$$
\frac{1}{C} \cdot\|u-v\|_{\mathbf{L}_{\mathrm{per}}^{1}} \leq J(u, v) \leq C \cdot\|u-v\|_{H_{\mathrm{per}}^{1}},
$$

with a constant $C$ uniformly valid on bounded subsets of $H_{\mathrm{per}}^{1}$.

Proof. We shall use the elementary bound

$$
|\arctan a-\arctan b| \cdot a^{2} \leq 4 \pi(|a|+|b|)|a-b|,
$$

valid for all $a, b \in \mathbb{R}$. In connection with the identity mapping $\psi(x)=x$ we now compute

$$
\begin{aligned}
J^{\psi}(u, v) & \leq \int_{0}^{1}\left\{|u(x)-v(x)|+2\left|\arctan u_{x}-\arctan v_{x}\right|\right\}\left(1+u_{x}^{2}\right) d x+\int_{0}^{1}\left|u_{x}^{2}-v_{x}^{2}\right| d x \\
& \leq\|u-v\|_{\mathbf{L}^{\infty}}\left\|1+u_{x}^{2}\right\|_{\mathbf{L}^{1}}+(8 \pi+1) \int_{0}^{1}\left|u_{x}+v_{x}\right|\left|u_{x}-v_{x}\right| d x \\
& \leq(8 \pi+3)\left(1+\|u\|_{H^{1}}+\|v\|_{H^{1}}\right) \cdot\|u-v\|_{H^{1}},
\end{aligned}
$$

proving the second inequality in (3.8).

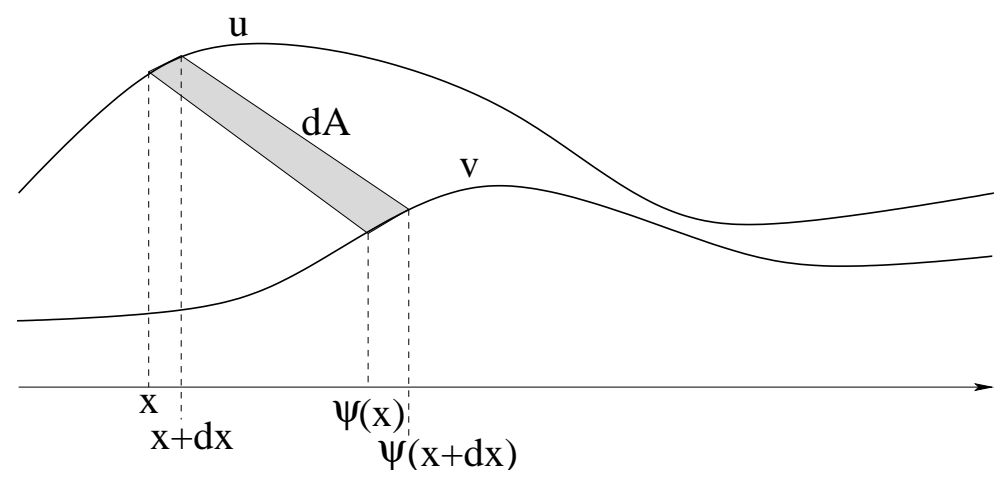

FIG. 1 
To achieve the first inequality, choose any $\psi \in \mathcal{F}$. For $x \in[0,1]$, call $\gamma^{x}$ the segment joining the point $P^{x}=(x, u(x))$ with $Q^{x}=(\psi(x), v(\psi(x)))$. Clearly, the union of all these segments covers the region between the graphs of $u$ and $v$. Moving the base point from $x$ to $x+d x$, the corresponding segments sweep an infinitesimal area $d A$ estimated by (fig. 1 )

$$
\begin{aligned}
|d A| & \leq\left|P^{x}-Q^{x}\right| \cdot\left(\left|d P^{x}\right|+\left|d Q^{x}\right|\right) \\
& \leq\left(|x-\psi(x)|^{2}+|u(x)-v(\psi(x))|^{2}+\right)^{1 / 2} \cdot\left[\left(1+u_{x}^{2}\right)^{1 / 2} d x+\left(1+v_{x}^{2}\right)^{1 / 2} \psi^{\prime}(x) d x\right] .
\end{aligned}
$$

Integrating over one period we obtain

$$
\begin{aligned}
& \int_{0}^{1}|u(x)-v(x)| d x \\
& \quad \leq \int_{0}^{1}\left(\left|x-\psi_{(t)}(x)\right|+\left|u(x)-v\left(\psi_{(t)}(x)\right)\right|\right) \cdot\left[\left(1+u_{x}^{2}(x)\right)+\left(1+v_{x}^{2}(\psi(x))\right)^{1 / 2} \psi_{(t)}^{\prime}(x)\right] d x \\
& \quad \leq\left(2+\|u\|_{H^{1}}+\|v\|_{H^{1}}\right) \cdot\left[J^{\psi}(u, v)+J^{\psi^{-1}}(v, u)\right] \leq C \cdot J(u, v),
\end{aligned}
$$

completing the proof of $(3.8)$.

Lemma 4. Let $\left(u_{n}\right)_{n \geq 1}$ be a Cauchy sequence for the distance $J$, uniformly bounded in the $H_{\mathrm{per}}^{1}$ norm. Then

(i) There exists a limit function $u \in H_{\mathrm{per}}^{1}$ such that $u_{n} \rightarrow u$ in $\mathbf{L}^{\infty}$ and the sequence of derivatives $u_{n, x}$ converges to $u_{x}$ in $L_{\mathrm{per}}^{p}$, for $1 \leq p<2$.

(ii) Let $\mu_{n}$ be the absolutely continuous measure having density $u_{n, x}^{2}$ w.r.t. Lebesgue measure. Then one has the weak convergence $\mu_{n} \rightarrow \mu$, for some measure $\mu$ whose absolutely continuous part has density $u_{x}^{2}$.

Proof. 1. By Lemma 3 we already know the convergence $u_{n} \rightarrow u$, for some limit function $u \in \mathbf{L}_{\mathrm{per}}^{1}$. By a Sobolev embedding theorem, all functions $u_{n}, u$ are uniformly Hölder continuous. This implies $\left\|u_{n}-u\right\|_{\mathbf{L}^{\infty}} \rightarrow 0$.

To establish the convergence of derivatives, we first show that the sequence of functions

$$
v_{n} \doteq \exp \left\{2 i \arctan u_{n, x}\right\}
$$

is compact in $\mathbf{L}_{\mathrm{per}}^{1}$. Indeed, fix $\varepsilon>0$. Then there exists $N$ such that $J\left(u_{m}, u_{n}\right)<\varepsilon$ for $m, n \geq N$. We can now approximate $u_{N}$ in $H_{\mathrm{per}}^{1}$ with a piecewise affine function $\tilde{u}_{N}$ such that $J\left(\tilde{u}_{N}, u_{N}\right) \leq \varepsilon$. By assumption, choosing suitable transport maps $\psi_{n}$ we obtain

$\int_{0}^{1}\left|\exp \left\{2 i \arctan u_{n, x}(x)\right\}-\exp \left\{2 i \arctan \tilde{u}_{N, x}\left(\psi_{n}(x)\right)\right\}\right| d x \leq 2 J\left(u_{n}, \tilde{u}_{N}\right) \leq 4 \varepsilon$

for all $n \geq N$. We now observe that all functions $x \mapsto \exp \left\{2 i \arctan \tilde{u}_{N, x}\left(\psi_{n}(x)\right)\right\}$ are uniformly bounded, piecewise constant with the same number of jumps: namely, the number of subintervals on which $\tilde{u}_{N}$ is affine. The set of all such functions is compact in $\mathbf{L}_{\text {per. }}^{1}$. This argument shows that the sequence $v_{n} \doteq \exp \left\{2 i \arctan u_{n, x}\right\}$ 
eventually remains in an $\varepsilon$-neighborhood of a compact subset of $\mathbf{L}_{\text {per }}^{1}$. Since $\varepsilon>0$ can be taken arbitrarily small, by possibly choosing a subsequence we obtain the strong convergence $v_{n} \rightarrow v$ for some $v \in \mathbf{L}_{\text {per }}^{1}$.

2. From the uniform $H^{1}$ bounds and the $\mathbf{L}^{1}$ convergence of the functions $v_{n}$, we now derive the $\mathbf{L}^{p}$ convergence of the derivatives. For a given $\varepsilon>0$, define

$$
M \doteq \sup _{n}\left\|u_{n}\right\|_{H_{\mathrm{per}}^{1}}, \quad A_{n} \doteq\left\{x \in[0,1] ; \quad\left|u_{n, x}(x)\right|>M / \varepsilon\right\}
$$

The above definitions imply

$$
\operatorname{meas}\left(A_{n}\right) \leq \varepsilon^{2}
$$

We now have

$$
\begin{gathered}
\left\|u_{m, x}-u_{n, x}\right\|_{\mathbf{L}^{p}} \leq\left(\int_{A_{n} \cup A_{m}}\left|u_{m, x}-u_{n, x}\right|^{p} d x\right)^{1 / p}+\left(\int_{[0,1] \backslash\left(A_{n} \cup A_{m}\right)}\left|u_{m, x}-u_{n, x}\right|^{p} d x\right)^{1 / p} \\
\quad I_{1}+I_{2} . \\
I_{1} \leq\left[\int_{A_{m} \cup A_{n}} 1 \cdot d x\right]^{(2-p) / 2 p} \cdot\left[\int_{A_{m} \cup A_{n}}\left(\left|u_{m, x}\right|+\left|u_{n, x}\right|\right)^{2} d x\right]^{1 / 2} \\
\leq \varepsilon^{(2-p) / p} \cdot 2 M .
\end{gathered}
$$

Next, choosing a constant $C_{\varepsilon}$ such that

$$
\left|e^{2 i \arctan a}-e^{2 i \arctan b}\right| \geq C_{\varepsilon}|a-b| \quad \text { whenever }|a|,|b| \leq M / \varepsilon,
$$

we obtain

$$
I_{2} \leq C_{\varepsilon}\left[\int\left|e^{2 i \arctan u_{m, x}}-e^{2 i \arctan u_{n, x}}\right|^{p} d x\right]^{1 / p} .
$$

Taking $\varepsilon>0$ small, we can make the right hand side of (3.13) as small as we like. On the other hand, choosing a subsequence such that $v_{\nu}=e^{2 i \arctan u_{\nu, x}}$ converges in $\mathbf{L}_{\text {per }}^{1}$, the right hand side of (3.154) approaches zero. Hence, for this subsequence,

$$
\limsup _{m, n \rightarrow \infty}\left\|u_{m, x}-u_{n, x}\right\|_{\mathbf{L}_{\mathrm{per}}^{p}}=0
$$

Since $u_{n} \rightarrow u$ uniformly, in this case we must have

$$
\left\|u_{n, x}-u_{x}\right\|_{\mathbf{L}_{\mathrm{per}}^{p}} \rightarrow 0
$$

We now observe that from any subsequence we can extract a further subsequence for which (3.15) holds. Therefore, the whole sequence $\left(u_{n, x}\right)_{n \geq 1}$ converges to $u_{x}$ in $\mathbf{L}_{\text {per }}^{p}$.

3. To establish (ii), we consider the sequence of measures having density $1+u_{n, x}^{2}$ w.r.t. Lebesgue measure. This sequence converges weakly, because our distance functional is stronger than the Kantorovich-Waserstein metric which induces the topology 
of weak convergence on spaces of measures. Therefore, $\mu_{n} \rightarrow \mu$ for some positive measure $\mu$.

Since the sequence $1+u_{n, x}$ converges to $1+u_{x}$ in $\mathbf{L}_{\text {per }}^{1}$, by possibly choosing a subsequence we achieve the pointwise convergence $u_{n, x}(x) \rightarrow u_{x}(x)$, for a.e. $x \in[0,1]$. For any $\varepsilon>0$, by Egorov's theorem we have the uniform convergence $u_{n, x}(x) \rightarrow u_{x}(x)$ for all $x \in[0,1] \backslash V_{\varepsilon}$, for some set with meas $\left(V_{\varepsilon}\right)<\varepsilon$. Since $\varepsilon>0$ can be taken arbitrarily small, this shows that the absolutely continuous part of the measure $\mu$ has density $u^{2}+u_{x}^{2}$ w.r.t. Lebesgue measure.

4. Continuity in time of the distance functional. Here and in the next section we examine how the distance functional $J(\cdot, \cdot)$ evolves in time, in connection with multi-peakon solutions of the Camassa-Holm equation (1.1). We first provide estimates valid on a time interval where no peakon interactions occur. Then we show that the distance functional is continuous across times of interaction. Since the number of peakons is locally finite, this will suffice to derive the basic estimates (1.17)-(1.18), in the case of multi-peakon solutions.

Lemma 5. Let $t \mapsto u(t) \in H_{\mathrm{per}}^{1}$ be a multi-peakon solution of (1.1). Assume that no peakon interactions occur within the interval $[0, \tau]$. Then

$$
J\left(u(s), u\left(s^{\prime}\right)\right) \leq C \cdot\left|s-s^{\prime}\right|, \quad \text { for all } s, s^{\prime} \in[0, \tau],
$$

for some constant $C$, uniformly valid as $u$ ranges on bounded subsets of $H_{\mathrm{per}}^{1}$.

Proof. Assume $0 \leq s<s^{\prime} \leq \tau$. By the assumptions, the solution $u=u(t, x)$ remains uniformly Lipschitz continuous on the time interval $[0, \tau]$. Therefore, for each $s \in[0, \tau]$ and $x \in \mathbb{R}$, the Cauchy problem

$$
\frac{d}{d t} \xi(t)=u(t, \xi(t)), \quad \xi(s)=x,
$$

determines a unique characteristic curve $t \mapsto \xi(t ; s, x)$ passing through the point $(s, x)$. Given $s^{\prime} \in[0, \tau]$, we can thus define a transportation plan by setting

$$
\psi(x) \doteq \xi\left(s^{\prime} ; s, x\right) .
$$

Of course, moving mass along the characteristics is the most natural thing to do. We then choose $\phi_{1}, \phi_{2}$ to be as large as possible, according to (3.4). Namely:

$$
\begin{aligned}
& \phi_{1}(x) \doteq \sup \left\{\theta \in[0,1] ; \theta \cdot\left(1+u_{x}^{2}(s, x)\right) \leq \psi^{\prime}(x) \cdot\left(1+u_{x}^{2}\left(s^{\prime}, \psi(x)\right)\right)\right\}, \\
& \phi_{2}(\psi(x)) \doteq \sup \left\{\theta \in[0,1] ; \quad \theta \cdot\left(1+u_{x}^{2}\left(s^{\prime}, \psi(x)\right)\right) \psi^{\prime}(x) \leq 1+u_{x}^{2}(s, x)\right\} .
\end{aligned}
$$

The cost of this plan is bounded by

$$
\begin{aligned}
J^{\psi}(u(s), & \left.u\left(s^{\prime}\right)\right) \leq \int_{0}^{1}\left\{\left|x-\xi\left(s^{\prime} ; s, x\right)\right|+\left|u(s, x)-u\left(s^{\prime}, \xi\left(s^{\prime} ; s, x\right)\right)\right|\right. \\
& \left.+\left|2 \arctan u_{x}(s, x)-2 \arctan u_{x}\left(s^{\prime}, \xi\left(s^{\prime} ; s, x\right)\right)\right|_{*}\right\}\left(1+u_{x}^{2}(s, x)\right) d x \\
& +\int_{0}^{1}\left(1-\phi_{1}(x)\right)\left(1+u_{x}^{2}(s, x)\right) d x \\
& +\int_{0}^{1}\left(1-\phi_{2}(\psi(x))\right)\left(1+u_{x}^{2}\left(s^{\prime}, \psi(x)\right)\right) \psi^{\prime}(x) d x
\end{aligned}
$$


To estimate the right hand side of (4.4), we first observe that, for all $u \in H_{\mathrm{per}}^{1}$,

$$
\begin{aligned}
\|u\|_{\mathbf{L}^{\infty}} & \leq \int_{0}^{1}|u(x)| d x+\int_{0}^{1}\left|u_{x}(x)\right| d x \\
& \leq\|u\|_{\mathbf{L}^{2}}+\left\|u_{x}\right\|_{\mathbf{L}^{2}} \leq 2\|u\|_{H_{\mathrm{per}}^{1}}=2\left(E^{u}\right)^{1 / 2} .
\end{aligned}
$$

Using (4.5) in (4.2) we obtain

$$
\left|\xi(s)-\xi\left(s^{\prime}\right)\right| \leq 2\left(E^{\bar{u}}\right)^{1 / 2} \cdot\left|s-s^{\prime}\right|
$$

Next, from the definition of the source term $P$ at (1.2) it follows

$$
\|P\|_{\mathbf{L}^{\infty}} \leq \frac{1}{2}\left\|e^{-|x|}\right\|_{\mathbf{L}^{1}(\mathbb{R})} \cdot\left\|u^{2}+\frac{u_{x}^{2}}{2}\right\|_{\mathbf{L}^{1}([0,1])} \leq\|u\|_{H_{\mathrm{per}}^{1}}^{2}=E^{u}
$$

Similarly,

$$
\left\|P_{x}\right\|_{\mathbf{L} \infty} \leq\|u\|_{H_{\mathrm{per}}^{1}}^{2}=E^{u} .
$$

Using (4.8) we obtain

$$
\begin{aligned}
\mid u\left(s^{\prime}, \xi\left(s^{\prime}\right)\right) & -u(s, \xi(s))\left|\leq \int_{s}^{s^{\prime}}\right| \frac{d}{d t} u(t, \xi(t)) \mid d t \\
& =\int_{s}^{s^{\prime}}\left|P_{x}(t, \xi(t))\right| d t \leq E^{\bar{u}} \cdot\left|s^{\prime}-s\right| .
\end{aligned}
$$

Concerning the term involving arctangents, recalling (1.3) we obtain

$$
\frac{d}{d t}\left[2 \arctan u_{x}(t, \xi(t, x))\right]=\frac{2}{1+u_{x}^{2}}\left[u^{2}-\frac{u_{x}^{2}}{2}-P\right]
$$

The bounds (4.7) and (4.9) thus yield

$$
\begin{aligned}
\left|2 \arctan u_{x}\left(s^{\prime}, \xi\left(s^{\prime}\right)\right)-2 \arctan u_{x}(s, \xi(s))\right|_{*} & \leq\left(2\|u\|_{\mathbf{L}^{\infty}}^{2}+1+2\|P\|_{\mathbf{L}^{\infty}}\right) \cdot\left|s^{\prime}-s\right| \\
& \leq\left(10 E^{\bar{u}}+1\right) \cdot\left|s^{\prime}-s\right| .
\end{aligned}
$$

This already provides a bound on the first integral on the right hand side of (4.4).

Next, call $I_{1}, I_{2}$ the last two integrals on the right hand side of (4.4). Notice that

$$
I_{1}+I_{2}=\int_{0}^{1}\left|\left(1+u_{x}^{2}(s, y)\right)-\xi_{y}\left(s^{\prime} ; s, y\right)\left(1+u_{x}^{2}\left(s^{\prime}, \xi\left(s^{\prime} ; s, y\right)\right)\right)\right| d y
$$

Indeed, $I_{1}+I_{2}$ measures the difference between the measure $\left(1+u_{x}^{2}\left(s^{\prime}, y\right)\right) d y$ and the push-forward of the measure $\left(1+u_{x}^{2}(s, x)\right) d x$ through the mapping $x \mapsto \xi\left(s^{\prime} ; s, x\right)$.

Since the push-forward of the measure $u_{x}^{2} d y$ satisfies the linear conservation law

$$
w_{t}+(u w)_{x}=0
$$


comparing (4.12) with (1.5) we deduce

$$
\begin{gathered}
\int_{0}^{1}\left|u_{x}^{2}(s, y)-\xi_{y}(s, y) u_{x}^{2}\left(s^{\prime}, \xi\left(s^{\prime} ; s, y\right)\right)\right| d y \leq \int_{s}^{s^{\prime}} \int_{0}^{1} 2\left|\left(u^{2}-P\right) u_{x}\right| d x d t \\
\leq 2 \int_{s}^{s^{\prime}}\left(\|u\|_{\mathbf{L}^{\infty}}^{2}+\|P\|_{\mathbf{L}^{\infty}}\right)\left\|u_{x}\right\|_{\mathbf{L}^{1}} d t \leq 2\left(4 E^{\bar{u}}+E^{\bar{u}}\right) E^{\bar{u}} \cdot\left|s^{\prime}-s\right|,
\end{gathered}
$$

because of (4.5), (4.7) and (1.15). Finally, we need to estimate the remaining terms, describing by how much the Lebesgue measure fails to be conserved by the transformation $x \mapsto \xi\left(s^{\prime} ; s, x\right)$. Observing that

$$
\frac{\partial}{\partial t} \xi_{y}(t, y)=u_{x}(t, \xi(t, y)) \xi_{y}(t, y), \quad \xi_{y}(0, y)=1
$$

we find

$$
\begin{gathered}
\int_{0}^{1}\left|1-\xi_{y}\left(s^{\prime} ; s, y\right)\right| d y \leq \int_{s}^{s^{\prime}} \int_{0}^{1}\left|\frac{\partial}{\partial t}\left[\xi_{y}(t, s, y)\right]\right| d y d t \\
\leq \int_{s}^{s^{\prime}} \int_{0}^{1} \xi_{y}(t ; s, y)\left|u_{x}(t, \xi(t ; s, y))\right| d y d t .
\end{gathered}
$$

To estimate the right hand side of (4.15), we use the decomposition $[0,1]=Y \cup Y^{\prime} \cup Y^{\prime \prime}$, where

$$
\begin{gathered}
Y \doteq\left\{y ; \quad \xi_{y}(t ; s, y) \in[(1 / 2), 2] \quad \text { for all } t \in\left[s, s^{\prime}\right]\right\}, \\
Y^{\prime} \doteq\left\{y ; \quad \xi_{y}(t ; s, y)<1 / 2 \text { for some } t \in\left[s, s^{\prime}\right]\right\}, \\
Y^{\prime \prime} \doteq\left\{y ; \quad \xi_{y}(t ; s, y)>2 \text { for some } t \in\left[s, s^{\prime}\right]\right\} .
\end{gathered}
$$

Integrating over $Y$ one finds

$$
\int_{s}^{s^{\prime}} \int_{Y} \xi_{y}(t ; s, y)\left|u_{x}(t, \xi(t ; s, y))\right| d y d t \leq 2 \int_{s}^{s^{\prime}}\left\|u_{x}(t)\right\|_{\mathbf{L}^{1}} d t \leq 2 E^{\bar{u} \cdot\left|s^{\prime}-s\right|}
$$

Next, if $y \in Y^{\prime}$ we define

$$
\tau(y) \doteq \inf \left\{t>s ; \quad \xi_{y}(t ; s, y)<1 / 2\right\} .
$$

Observe that $y \in Y^{\prime}$ implies

$$
\int_{s}^{\tau(y)}\left|u_{x}(t, \xi(t ; s, y))\right| d t \geq \ln 2
$$

Therefore

$$
\begin{aligned}
\int_{Y^{\prime}} d y & \leq \frac{1}{\ln 2} \int_{Y^{\prime}}\left[\int_{s}^{\tau(y)}\left|u_{x}(t, \xi(t ; s, y))\right| d t\right] d y \\
& \leq \frac{2}{\ln 2} \int_{s}^{s^{\prime}} \int_{0}^{1}\left|u_{x}(t, x)\right| d x d t \leq 4 E^{\bar{u}} \cdot\left|s^{\prime}-s\right| .
\end{aligned}
$$

The estimate for the integral over $Y^{\prime \prime}$ is entirely analogous, Indeed, the push-forward of the Lebesgue measure along characteristic curves from $t=s$ to $t=s^{\prime}$ satisfies 
exactly the same type of estimates as the pull-back of the Lebesgue measure from $t=s^{\prime}$ to $t=s$. All together, these three estimates imply

$$
\int_{Y \cup Y^{\prime} \cup Y^{\prime \prime}}\left|1-\xi_{y}\left(s^{\prime} ; s, y\right)\right| d y \leq 10 E^{\bar{u}} \cdot\left|s^{\prime}-s\right|
$$

Putting together the estimates (4.6), (4.9), (4.10), (4.3) and (4.17), the distance in (4.4) can be estimated by

$$
J^{\psi}\left(u(s), u\left(s^{\prime}\right)\right) \leq\left[2\left(1+E^{\bar{u}}\right)+E^{\bar{u}}+\left(10 E^{\bar{u}}+1\right)+10\left(E^{\bar{u}}\right)^{2}+10 E^{\bar{u}}\right] \cdot\left|s^{\prime}-s\right| .
$$

This establishes (4.1).

According to Lemma 5, as long as no peakon interactions occur, the map $t \mapsto u(t)$ remains uniformly Lipschitz continuous w.r.t. our distance functional, with a Lipschitz constant that depends only on the total energy $E^{\bar{u}}$. Since interactions can occur only at isolated times, to obtain a global Lipschitz estimate it suffices to show that trajectories are continuous (w.r.t. the distance $J$ ) also at interaction times.

Lemma 6. Assume that the multi-peakon solution $u(\cdot)$ contains two or more peakons which interact at a time $\tau$. Then

$$
\lim _{h \rightarrow 0+} J(u(\tau-h), u(\tau+h))=0 .
$$

Proof. To fix the ideas, call $x=\bar{q}$ the place where the interaction occurs, and let $p_{1}, \ldots, p_{k}$ be the strengths of the peakons that interact at time $\tau$. We here assume that $0<\bar{q}<1$. The case where two or more groups of peakons interact exactly at the same time $\tau$, within the interval $[0,1]$, can be treated similarly.

For $|t-\tau| \leq h$, call $\xi^{-}(t), \xi^{+}(t)$ respectively the position of the smallest and largest characteristic curves passing through the point $(\tau, \bar{q})$, as in $(2.5)$. We observe that $u$ is Lipschitz continuous in a neighborhood of each point $(\tau, x)$, with $x \neq \bar{q}$. Hence, for $x \in[0,1] \backslash\{\bar{q}\}$ there exists a unique characteristic curve $t \mapsto \xi(t ; \tau, x)$ passing through $x$ at time $\tau$. For a fixed $h>0$, the transport map $\psi$ is defined as follows. Consider the intervals $I_{-h} \doteq\left[\xi^{-}(\tau-h), \xi^{+}(\tau-h)\right]$ and $I_{h} \doteq\left[\xi^{-}(\tau+h), \xi^{+}(\tau+h)\right]$. On the complement $[0,1] \backslash I_{-h}$ we define

$$
\psi(\xi(\tau-h ; \tau, x))=\xi(\tau+h ; \tau, x),
$$

so that transport is performed along characteristic curves. It now remains to extends $\psi$ as a map from $I_{-h}$ onto $I_{h}$. Toward this goal, we recall that our construction of multi-peakon solutions in Section 2 was specifically designed in order to achieve the identity

$$
e_{(\tau, \bar{q})}=\lim _{h \rightarrow 0+} \int_{I_{-h}} u_{x}^{2}(\tau-h, x) d x=\lim _{h \rightarrow 0+} \int_{I_{h}} u_{x}^{2}(\tau+h, x) d x .
$$

For $h>0$ we introduce the quantities

$$
E(-h) \doteq \int_{I_{-h}}\left(1+u_{x}^{2}(\tau-h, x)\right) d x, \quad E(h) \doteq \int_{I_{h}}\left(1+u_{x}^{2}(\tau+h, x)\right) d x,
$$




$$
e(h) \doteq 2 \min \{E(-h), E(h)\}-\max \{E(-h), E(h)\} .
$$

Notice that (4.20) implies $e(h)>0$ and

$$
E(-h) \rightarrow e_{(\tau, \bar{q})}, \quad E(h) \rightarrow e_{(\tau, \bar{q})}, \quad e(h) \rightarrow e_{(\tau, \bar{q})},
$$

as $h \rightarrow 0+$. Consider the point $x^{*}=x^{*}(h)$ inside the interval $I_{-h}=\left[\xi^{-}(\tau-h), \xi^{+}(\tau-\right.$ $h)$ ], implicitly defined by

$$
\int_{\xi^{-}(\tau-h)}^{x^{*}}\left(1+u_{x}^{2}(x)\right) d x=e(h) .
$$

For $x \in\left[\xi^{-}(\tau-h), x^{*}\right]$ we define $\psi(x)$ as the unique point such that

$$
\int_{\xi^{-}(\tau+h)}^{\psi(x)}\left(1+u_{x}^{2}(\tau+h, x)\right) d x=\int_{\xi^{-}(\tau-h)}^{x}\left(1+u_{x}^{2}(\tau-h, x)\right) d x .
$$

We then extend $\psi$ as an affine map from $\left[x^{*}, \xi^{+}(\tau-h)\right]$ onto $\left[\psi\left(x^{*}\right), \xi^{+}(\tau+h)\right]$, namely

$$
\psi\left(\theta \cdot \xi^{+}(\tau-h)+(1-\theta) \cdot x^{*}\right)=\theta \cdot \xi^{+}(\tau+h)+(1-\theta) \cdot \psi\left(x^{*}\right) \quad \theta \in[0,1] .
$$

Finally, we prolong $\psi$ to the whole real line according to (3.3). As usual, the 1periodic functions $\phi_{1}, \phi_{2}$ are then chosen to be as large as possible, according to (3.4). As $h \rightarrow 0+$, we claim that the following quantity approaches zero:

$$
\begin{aligned}
& J^{\psi}(u(\tau-h), u(\tau+h)) \\
& =\int_{0}^{1} d \diamond\left(\left(x, u(\tau-h, x), 2 \arctan u_{x}(\tau-h, x)\right)\right. \\
& \left.\left(\psi(x), u(\tau+h, \psi(x)), 2 \arctan \tilde{u}_{x}(\tau+h, \psi(x))\right)\right) \\
& \quad \cdot \phi_{1}(x)\left(1+u_{x}^{2}(\tau-h, x)\right) d x \\
& \quad+\int_{0}^{1}\left(1-\phi_{1}(x)\right)\left(1+u_{x}^{2}(\tau-h, x)\right) d x \\
& \quad+\int_{0}^{1}\left(1-\phi_{2}(\psi(x))\right)\left(1+u_{x}^{2}(\tau+h, \psi(x))\right) \psi^{\prime}(x) d x .
\end{aligned}
$$

It is clear that the restriction of all the above integrals to the complement $[0,1] \backslash I_{-h}$ approaches zero as $h \rightarrow 0$. We now prove that their restriction to $I_{-h}$ also vanishes in the limit. As $h \rightarrow 0+$, for $x \in I_{-h}$ we have

$d^{\diamond}\left(\left(x, u(\tau-h, x), 2 \arctan u_{x}(\tau-h, x)\right),\left(\psi(x), u(\tau+h, \psi(x)), 2 \arctan u_{x}(\tau+h, \psi(x))\right)\right) \rightarrow 0$,

because all points approach the same limit $(\bar{q}, u(\tau, \bar{q}), \pi)$. The first integral in (4.23) thus approaches zero as $h \rightarrow 0+$.

Concerning the last two integrals, by (4.22) it follows

$$
\phi_{1}(x)=\phi_{2}(\psi(x))=1 \quad \text { for all } x \in\left[\xi^{-}(\tau-h), x^{*}\right] .
$$


Moreover, our choice of $x^{*}$ implies

$$
\begin{aligned}
& \int_{x^{*}}^{\xi^{+}(\tau-h)}\left(1+u_{x}^{2}(\tau-h, x)\right) d x+\int_{\psi\left(x^{*}\right)}^{\xi^{+}(\tau+h)}\left(1+u_{x}^{2}(\tau+h, \psi(x))\right) \psi^{\prime}(x) d x \\
& \leq 2 \max \{E(-h), E(h)\}-2 \min \{E(-h), E(h)\} .
\end{aligned}
$$

By (4.21), as $h \rightarrow 0+$ the right hand side of (4.24) approaches zero. Hence the same holds for the last two integrals in (4.23). This completes the proof of the lemma.

5. Continuity w.r.t. the initial data. We now consider two distinct solutions and study how the distance $J(u(t) v(t))$ evolves in time. To fix the ideas, let $t \mapsto u(t)$ and $t \mapsto v(t)$ be two multi-peakon solutions of (1.1), and assume that no interaction occurs within a given time interval $[0, T]$. In this case, the functions $u, v$ remain Lipschitz continuous. We can thus define the characteristic curves $t \mapsto \xi(t, y)$ and $t \mapsto \zeta(t, \tilde{y})$ as the solutions to the Cauchy problems

$$
\begin{array}{ll}
\dot{\xi}=u(t, \xi), & \xi(0)=y, \\
\dot{\zeta}=v(t, \zeta), & \zeta(0)=\tilde{y},
\end{array}
$$

respectively. Let now $\psi_{(0)} \in \mathcal{F}$ be any transportation plan at time $t=0$. For each $t \in[0, T]$ we can define a transportation plan $\psi_{(t)} \in \mathcal{F}$ by setting

$$
\psi_{(t)}(\xi(t, y)) \doteq \zeta\left(t, \psi_{(0)}(y)\right) .
$$

The corresponding functions $\phi_{1}^{(t)}, \phi_{2}^{(t)}$ are then defined according to (3.4), namely

$$
\begin{gathered}
\phi_{1}^{(t)}(x) \doteq \sup \left\{\theta \in[0,1] ; \theta \cdot\left(1+u_{x}^{2}(t, x)\right) \leq\left(1+v_{x}^{2}\left(t, \psi_{(t)}(x)\right)\right) \psi_{(t)}^{\prime}(x)\right\}, \\
\phi_{2}^{(t)}\left(\psi_{(t)}(x)\right) \doteq \sup \left\{\theta \in[0,1] ; 1+u_{x}^{2}(t, x) \geq \theta \cdot\left(1+v_{x}^{2}\left(t, \psi_{(t)}(x)\right)\right) \psi_{(t)}^{\prime}(x)\right\} .
\end{gathered}
$$

If initially the point $y$ is mapped into $\tilde{y} \doteq \psi_{(0)}(y)$, then at a later time $t>$ 0 the point $\xi(t, y)$ along the $u$-characteristic starting from $y$ is sent to the point $\zeta(t, \tilde{y})$ along the $v$-characteristic starting from $\tilde{y}$. We thus transport mass from the point $\left(\xi(t, y), u(t, \xi(t, y)), 2 \arctan u_{x}(t, \xi(t, y))\right)$ to the corresponding point $\left(\zeta(t, \tilde{y}), v(t, \zeta(t, \tilde{y})), 2 \arctan v_{x}(t, \zeta(t, \tilde{y}))\right)$,

In the following, our main goal is to provide an upper bound on the time derivative of the function

$$
\begin{aligned}
J^{\psi(t)}(u(t), v(t)) \doteq \int_{0}^{1} d \diamond\left(\left(x, u(t, x), 2 \arctan u_{x}(t, x)\right)\right. \\
\left.\quad\left(\psi_{(t)}(x), v\left(t, \psi_{(t)}(x)\right), 2 \arctan v_{x}\left(t, \psi_{(t)}(x)\right)\right)\right) \cdot \phi_{1}^{(t)}(x)\left(1+u_{x}^{2}(t, x)\right) d x \\
+\int_{0}^{1}\left(1-\phi_{1}^{(t)}(x)\right)\left(1+u_{x}^{2}(t, x)\right) d x \\
+\int_{0}^{1}\left(1-\phi_{2}^{(t)}\left(\psi_{(t)}(x)\right)\right)\left(1+v_{x}^{2}\left(t, \psi_{(t)}(x)\right)\right) \psi_{(t)}^{\prime}(x) d x
\end{aligned}
$$


Differentiating the right hand side of (5.3) one obtains several terms, due to

- Changes in the distance $d \diamond$ between the points $\left(\xi, u, 2 \arctan u_{x}\right)$ and $\left(\zeta, v, 2 \arctan v_{x}\right)$.

- Changes in the base measures $\left(1+u_{x}^{2}\right) d x$ and $\left(1+v_{x}^{2}\right) d x$.

Throughout the following, by $\mathcal{O}(1)$ we denote a quantity which remains uniformly bounded as $u, v$ range in bounded subsets of $H_{\mathrm{per}}^{1}$. Using the elementary estimate

$$
|u-v| \leq(1+|u|+|v|) \min \{|u-v|, 1\},
$$

we begin by deriving the bound

$$
\begin{aligned}
I_{1} & \doteq \int_{0}^{1} \frac{d}{d t}\left|x-\psi_{(t)}(x)\right| \cdot \phi_{1}^{(t)}(x)\left(1+u_{x}^{2}(t, x)\right) d x \\
& \leq \int_{0}^{1}\left|u(t, x)-v\left(t, \psi_{(t)}(x)\right)\right| \cdot \phi_{1}^{(t)}(x)\left(1+u_{x}^{2}(t, x)\right) d x \\
& \leq\left(1+\|u(t)\|_{\mathbf{L}^{\infty}}+\|v(t)\|_{\mathbf{L}^{\infty}}\right) \cdot J^{\psi_{(t)}}(u(t), v(t)) \\
& =\mathcal{O}(1) \cdot J^{\psi_{(t)}}(u(t), v(t)) .
\end{aligned}
$$

Here and in the sequel, the time derivative is computed along characteristics.

Next, recalling the basic equation (1.1), we consider

$$
\begin{aligned}
I_{2} & \doteq \int_{0}^{1} \frac{d}{d t}\left|u(t, x)-v\left(t, \psi_{(t)}(x)\right)\right| \cdot \phi_{1}^{(t)}(x)\left(1+u_{x}^{2}(t, x)\right) d x \\
& \leq \int_{0}^{1}\left|P_{x}^{u}(t, x)-P_{x}^{v}\left(t, \psi_{(t)}(x)\right)\right| \cdot\left(1+u_{x}^{2}(t, x)\right) d x .
\end{aligned}
$$

In the spatially periodic case, by (1.2) and (2.2) we can write the source terms $P_{x}^{u}$, $P_{x}^{v}$ as

$$
\begin{aligned}
P_{x}^{u}(t, x) & =\frac{1}{2} \int_{x-1}^{x} \chi^{\prime}(x-y) \cdot\left[u^{2}(t, y)+\frac{u_{x}^{2}(t, y)}{2}\right] d y \\
P_{x}^{v}\left(t, \psi_{(t)}(x)\right)= & \frac{1}{2} \int_{0}^{1} \chi^{\prime}\left(\psi_{(t)}(x)-\tilde{y}\right) \cdot v^{2}(t, \tilde{y}) d \tilde{y} \\
& \quad+\int_{x-1}^{x} \chi^{\prime}\left(\psi_{(t)}(x)-\psi_{(t)}(y)\right) \cdot \frac{v_{x}^{2}\left(t, \psi_{(t)}(y)\right)}{4} \psi_{(t)}^{\prime}(y) d y
\end{aligned}
$$

where, according to $(2.2)$,

$$
\chi^{\prime}(x)=\frac{e^{x}-e^{1-x}}{e-1} \quad 0<x<1, \quad \chi^{\prime}(x)=\chi^{\prime}(x+1) \quad x \in \mathbb{R} .
$$

In the next computation, we use the estimate

$$
\int_{0}^{1}\left|\left(1+u_{x}^{2}(y)\right)-\left(1+v_{x}^{2}(\psi(y))\right) \psi^{\prime}(y)\right| d y=\mathcal{O}(1) \cdot J^{\psi}(u, v) .
$$


which holds because of the last two terms in the definition (3.6). Observing that $\chi^{\prime}$ is Lipschitz continuous on the open interval $] 0,1[$, we now compute (omitting explicit references to the time $t$ )

$$
\begin{aligned}
\mid P_{x}^{u}(x)- & P_{x}^{v}(\psi(x))\left|\leq \frac{1}{2} \int_{0}^{1}\right| \chi^{\prime}(x-y) \cdot u^{2}(y)-\chi^{\prime}(\psi(x)-y) \cdot v^{2}(y) \mid d y \\
+ & \mathcal{O}(1) \cdot \int_{x-1}^{x}|x-y-(\psi(x)-\psi(y))| \cdot \frac{v_{x}^{2}(\psi(y))}{2} \psi^{\prime}(y) d y \\
+ & \frac{1}{4}\left|\int_{x-1}^{x} \chi^{\prime}(x-y)\left(u_{x}^{2}(y)-v_{x}^{2}(\psi(y)) \psi^{\prime}(y)\right) d y\right| \\
= & \mathcal{O}(1) \cdot\left(|x-\psi(x)|+|| u^{2}-v^{2} \|_{\mathbf{L}^{1}}\right) \\
& +\mathcal{O}(1) \cdot\left(|x-\psi(x)|+\int_{x-1}^{x}|y-\psi(y)| \cdot \frac{v_{x}^{2}(\psi(y))}{2} \psi^{\prime}(y) d y\right) \\
& +\mathcal{O}(1) \cdot\left(J \psi(u, v)+\left|\int_{x-1}^{x} \chi^{\prime}(x-y) \cdot\left[\psi^{\prime}(y)-1\right] d y\right|\right) \\
= & \mathcal{O}(1) \cdot|x-\psi(x)|+\mathcal{O}(1) \cdot J^{\psi}(u, v)+\mathcal{O}(1) \\
& \quad \cdot\left(|x-\psi(x)|+\int_{x-1}^{x} \chi^{\prime \prime}(x-y) \cdot[\psi(y)-y] d y\right) \\
= & \mathcal{O}(1) \cdot|x-\psi(x)|+\mathcal{O}(1) \cdot J^{\prime}(u, v) .
\end{aligned}
$$

Integrating over one period we conclude

$$
I_{2}=\mathcal{O}(1) \cdot J^{\psi}(u(t), v(t)) .
$$

For future use, we observe that a computation entirely similar to (5.9) yields

$$
\left|P_{x}^{u}(x)-P_{x}^{v}(\psi(x))\right|=\mathcal{O}(1) \cdot|x-\psi(x)|+\mathcal{O}(1) \cdot J^{\psi}(u, v) .
$$

Next, we look at the term

$$
I_{3} \doteq \int_{0}^{1} \frac{d}{d t}\left|2 \arctan u_{x}(t, x)-2 \arctan v_{x}\left(t, \psi_{(t)}(x)\right)\right| \cdot \phi_{1}^{(t)}(x)\left(1+u_{x}^{2}(t, x)\right) d x
$$

Along a characteristic, according to (1.3) one has

$$
\frac{d}{d t} 2 \arctan u_{x}(t, \xi(t))=\frac{2}{1+u_{x}^{2}}\left[u^{2}-\frac{u_{x}^{2}}{2}-P^{u}\right] .
$$

Call $\theta^{u} \doteq 2 \arctan u_{x}, \theta^{v} \doteq 2 \arctan v_{x}$, so that

$$
\frac{1}{1+u_{x}^{2}}=\cos ^{2} \frac{\theta^{u}}{2}, \quad \frac{u_{x}}{1+u_{x}^{2}}=\frac{1}{2} \sin \theta^{u}, \quad \frac{u_{x}^{2}}{1+u_{x}^{2}}=\sin ^{2} \frac{\theta^{u}}{2} .
$$

We now have

$$
\begin{aligned}
\int_{0}^{1} & \left(1+u_{x}^{2}(x)\right) \cdot\left|\frac{u_{x}^{2}(x)}{1+u_{x}^{2}(x)}-\frac{v_{x}^{2}(\psi(x))}{1+v_{x}^{2}(\psi(x))}\right| d x \\
& =\int_{0}^{1}\left(1+u_{x}^{2}(x)\right) \cdot\left|\sin ^{2} \frac{\theta^{u}(x)}{2}-\sin ^{2} \frac{\theta^{v}(\psi(x))}{2}\right| d x \\
& \leq \int_{0}^{1}\left(1+u_{x}^{2}(x)\right) \cdot\left|\theta^{u}(x)-\theta^{v}(\psi(x))\right| d x \\
& =\mathcal{O}(1) \cdot J(u, v) .
\end{aligned}
$$


Next, using (5.11) we compute

$$
\begin{aligned}
& \int_{0}^{1}\left(1+u_{x}^{2}(x)\right) \cdot \mid\left|\frac{u^{2}(x)-P^{u}(x)}{1+u_{x}^{2}(x)}-\frac{v^{2}(\psi(x))-P^{v}(\psi(x))}{1+v_{x}^{2}(\psi(x))}\right| d x \\
& \leq \int_{0}^{1}\left|u^{2}(x)-v^{2}(\psi(x))\right| d x+\int_{0}^{1}\left|P^{u}(x)-P^{v}(\psi(x))\right| d x \\
& \quad \quad+\mathcal{O}(1) \cdot \int_{0}^{1}\left|\frac{1}{1+u_{x}^{2}(x)}-\frac{1}{1+v_{x}^{2}(\psi(x))}\right| \cdot\left(1+u_{x}^{2}(x)\right) d x \\
&=\mathcal{O}(1) \cdot J^{\psi}(u, v),
\end{aligned}
$$

where the last term was estimated by observing that

$$
\left|\frac{1}{1+u_{x}^{2}}-\frac{1}{1+v_{x}^{2}}\right| \leq\left|2 \arctan u_{x}-2 \arctan v_{x}\right|_{*} .
$$

Putting together all previous estimates we conclude

$$
I_{1}+I_{2}+I_{3}=\mathcal{O}(1) \cdot J \psi(u, v) .
$$

To complete the analysis, we have to consider the terms due to the change in base measures. From (1.5) it follows that the production of new mass in the base measures is described by the balance laws

$$
\left\{\begin{array}{c}
\left(1+u_{x}^{2}\right)_{t}+\left[u\left(1+u_{x}^{2}\right)\right]_{x}=\left[2 u^{2}+1-2 P^{u}\right] u_{x} \doteq f^{u}, \\
\left(1+v_{x}^{2}\right)_{t}+\left[v\left(1+v_{x}^{2}\right)\right]_{x}=\left[2 v^{2}+1-2 P^{v}\right] v_{x} \doteq f^{v} .
\end{array}\right.
$$

This leads us to consider two further integrals $I_{4}, I_{5}$ :

$$
\begin{aligned}
I_{4}= & \int_{0}^{1} d \diamond \\
& \cdot\left(x, u(x), 2 \arctan u_{x}(x)\right),\left(\psi(x), v(\psi(x)), 2 \arctan v_{x}(\psi(x))\right) \\
= & \mathcal{O}(1) \cdot \int_{0}^{1} d \diamond\left(\left(x, u(x), 2 \arctan u_{x}(x)\right),\left(\psi(x), v(\psi(x)), 2 \arctan v_{x}(\psi(x))\right)\right. \\
= & \mathcal{O}(1) \cdot J^{u}\left(1+u_{x}^{2}(x)\right) d x \\
I_{5}= & \int_{0}^{1}\left|\left[2 u^{2}(x)+1-2 P^{u}(x)\right] u_{x}(x)-\left[2 v^{2}(\psi(x))+1-2 P^{v}(\psi(x))\right] v_{x}(\psi(x)) \psi^{\prime}(x)\right| d x \\
\leq & 2 \int_{0}^{1}\left\{\left|u^{2}(x)-v^{2}(\psi(x))\right|+\left|P^{u}(x)-P^{v}(\psi(x))\right|\right\}\left|u_{x}(x)\right| d x \\
& +\int_{0}^{1}\left|2 v^{2}(\psi(x))+1-2 P^{v}(\psi(x))\right| \cdot\left|u_{x}(x)-v_{x}(\psi(x)) \psi^{\prime}(x)\right| d x \\
= & I_{5}^{\prime}+I_{5}^{\prime \prime}
\end{aligned}
$$


Using (5.11) we easily obtain

$$
\begin{aligned}
I_{5}^{\prime} & \leq \int_{0}^{1}\left\{\left|u^{2}(x)-v^{2}(\psi(x))\right|+\left|P^{u}(x)-P^{v}(\psi(x))\right|\right\}\left(1+u_{x}^{2}(x)\right) d x \\
& =\mathcal{O}(1) \cdot J \psi(u, v) .
\end{aligned}
$$

On the other hand, recalling (5.13) and using the change of variable $y=\psi(x), x=$ $\psi^{-1}(y)$, we find

$$
\begin{aligned}
I_{5}^{\prime \prime}= & \mathcal{O}(1) \cdot \int_{0}^{1}\left|u_{x}(x)-v_{x}(\psi(x)) \psi^{\prime}(x)\right| d x \\
= & \mathcal{O}(1) \cdot \int_{0}^{1}\left|\frac{u_{x}(x)}{1+u_{x}^{2}(x)}-\frac{v_{x}(\psi(x))}{1+v_{x}^{2}(\psi(x))}\right|\left(1+u_{x}^{2}(x)\right) d x \\
& \quad+\mathcal{O}(1) \cdot \int_{0}^{1}\left|v_{x}(\psi(x)) \psi^{\prime}(x)\right| \cdot\left|\frac{1+u_{x}^{2}(x)}{\left(1+v_{x}^{2}(\psi(x))\right) \psi^{\prime}(x)}-1\right| \\
& \quad \mathcal{O}(1) \cdot J \psi(u, v)+\int_{0}^{1}\left|\left(1+u_{x}^{2}(x)\right)-\left(1+v_{x}^{2}(\psi(x))\right) \psi^{\prime}(x)\right| d x \\
= & \mathcal{O}(1) \cdot J(u, v) .
\end{aligned}
$$

All together, the previous estimates show that

$$
\frac{d}{d t} J^{\psi_{(t)}}(u(t), v(t)) \leq I_{1}+I_{2}+I_{3}+I_{4}+I_{5}^{\prime}+I_{5}^{\prime \prime}=\mathcal{O}(1) \cdot J^{\psi_{(t)}}(u(t), v(t)),
$$

where $\mathcal{O}(1)$ denotes a quantity which remains uniformly bounded as $u, v$ range on bounded sets of $H_{\mathrm{per}}^{1}$. As an immediate consequence we obtain

LEMMA 7. Let $t \mapsto u(t), t \mapsto v(t)$ be two conservative, spatially periodic multipeakon solutions, as in Lemma 2. Then there exists a constant $\kappa$, depending only on $\max \left\{\|u\|_{H_{\mathrm{per}}^{1}},\|v\|_{H_{\mathrm{per}}^{1}}\right\}$, such that

$$
J(u(t), v(t)) \leq e^{\kappa|t-s|} \cdot J(u(s), v(s)) \quad s, t \in \mathbb{R} .
$$

Proof. For $t>s$ the estimate (5.23) follows from (5.22), taking the infimum among all transportation plans $\psi_{(s)}$ at time $s$. The case $t<s$ is obtained simply by observing that the Camassa-Holm equations are time-reversible.

6. Proof of the main theorems. Thanks to the analysis in the previous sections, we now all the ingredients toward a proof of Theorems 1 and 2 .

The estimates in (1.16) follow from Lemma 3. Given an initial data $\bar{u} \in H_{\text {per }}^{1}$, to construct the solution of the Camassa-Holm equation we consider a sequence of multipeakons $\bar{u}_{n}$, converging to $\bar{u}$ in $H_{\text {per }}^{1}$. Then we consider the corresponding solutions $t \mapsto u_{n}(t)$, defined for all $n \geq 1$ and $t \in \mathbb{R}$. This is possible because of Lemmas 1 and 2 . 
We claim that the sequence $u_{n}(t)$ is Cauchy in $L_{\text {per }}^{2}$. Indeed, by Lemma 3 and Lemma 5,

$$
\begin{aligned}
\left\|u_{m}(t)-u_{n}(t)\right\|_{\mathbf{L}_{\mathrm{per}}^{1}} \leq C \cdot J\left(u_{m}(t), u_{n}(t)\right) & \leq C \cdot e^{\kappa|t|} J\left(u_{m}(0), u_{n}(0)\right) \\
& \leq C^{2} \cdot e^{\kappa|t|}\left\|u_{m}(t)-u_{n}(t)\right\|_{H_{\mathrm{per}}^{1}}
\end{aligned}
$$

Therefore, $u_{n}(t) \rightarrow u(t)$ in $\mathbf{L}_{\text {per }}^{1}$, for some function $u: \mathbb{R} \mapsto H_{\text {per }}^{1}$. By interpolation, the convergence $u_{n} \rightarrow u$ also holds in all spaces $\mathbf{L}_{\text {per }}^{p}, 1 \leq p \leq \infty$. The continuity estimates (1.17)-(1.18) now follow by passing to the limit in Lemma 5 and 6 .

It remains to show that the limit function $u(\cdot)$ is actually a solution to the Camassa-Holm equation and its energy $E(t)$ in (1.15) is a.e. constant. Toward these goals, we observe that all solutions $u_{n}$ are Lipschitz continuous with the same Lipschitz constant, as maps from $\mathbb{R}$ into $L_{\text {per }}^{2}$. Indeed

$$
\left\|u_{n, t}\right\|_{\mathbf{L}_{\text {per }}^{2}} \leq\left\|u_{n}\right\|_{\mathbf{L}^{\infty}} \cdot\left\|u_{n, x}\right\|_{\mathbf{L}_{\text {per }}^{2}}+\left\|\frac{1}{2} e^{-|x|}\right\|_{\mathbf{L}^{2}} \cdot\left\|u_{n}^{2}+\frac{u_{n, x}^{2}}{2}\right\|_{\mathbf{L}_{\text {per }}^{1}} .
$$

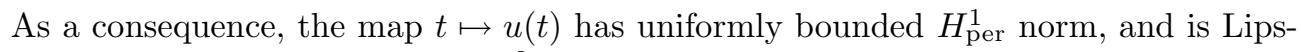
chitz continuous with values in $L_{\text {per }}^{2}$. In particular, $u$ is uniformly Hölder continuous as a function of $t, x$ and the convergence $u_{m}(t, x) \rightarrow u(t, x)$ holds uniformly for $t$ in bounded sets. Moreover, since $\mathbf{L}_{\text {per }}^{2}$ is a reflexive space, the time derivative $u_{t}(t) \in \mathbf{L}_{\text {per }}^{2}$ is well defined for a.e. $t \in \mathbb{R}$.

We now observe that, for each $n \geq 1$, both sides of the equality

$$
\frac{d}{d t} u_{n}=-u_{n} u_{n, x}-P_{x}^{u_{n}}
$$

are continuous as functions from $\mathbb{R}$ into $\mathbf{L}_{\text {per }}^{1}$, and the identity holds at every time $t \in \mathbb{R}$, with the exception of the isolated times where a peakon interaction occurs.

At any time $t$ where no peakon interaction occur in the solution $u_{n}$, we define $\mu_{t}^{(n)}$ to be the measure with density $u_{n}^{2}(t, \cdot)+\frac{1}{2} u_{n, x}(t, \cdot)$ w.r.t. Lebesgue measure. By Lemmas 5 and 4 , the map $t \mapsto \mu_{t}^{(n)}$ can be extended by weak continuity to all times $t \in \mathbb{R}$. We can now redefine

$$
P^{u_{n}}(t, x) \doteq \int \frac{1}{2} e^{-|x-y|} d \mu_{t}^{(n)}(y), \quad P^{u}(t, x) \doteq \int \frac{1}{2} e^{-|x-y|} d \mu_{t}(y),
$$

where $\mu_{t}$ is the weak limit of the measures $\mu_{t}^{(n)}$. Because of the convergence $J\left(u_{n}(t), u(t)\right) \rightarrow 0$, by Lemma 4 the map $t \mapsto \mu_{t}$ is well defined and continuous w.r.t. the weak topology of measures. Using again Lemma 4, we can take the limit of (6.2) as $n \rightarrow \infty$, and obtain the identity (1.14), for every $t \in \mathbb{R}$ and $P=P^{u}$ defined by $(6.3)$.

For each $n$, the total energy $\left.\left.\mu_{t}^{(n)}(] 0,1\right]\right)=E^{\bar{u}_{n}}$ is constant in time and converges to $E^{\bar{u}}$ as $n \rightarrow \infty$. Therefore we also have

$$
\left.\left.\mu_{t}(] 0,1\right]\right)=E^{\bar{u}} \doteq \int_{0}^{1}\left[\bar{u}^{2}(x)+\bar{u}_{x}^{2}(x)\right] d x \quad t \in \mathbb{R} .
$$

To complete the proof of Theorem 1, it now only remains to prove that the measure $\mu_{t}$ is absolutely continuous with density $u^{2}(t, \cdot)+\frac{1}{2} u_{x}^{2}(t, \cdot)$ w.r.t. Lebesgue measure, for a.e. time $t \in \mathbb{R}$. 
In this direction, we recall that, by (1.5), the function $w \doteq u_{n, x}^{2}$ satisfies the linear transport equation with source

$$
w_{t}+(u w)_{x}=\left(u_{n}^{2}-P^{u_{n}}\right) u_{n, x} .
$$

Moreover, along any characteristic curve $t \mapsto \xi(t)$ by (1.3) one has

$$
\frac{d}{d t}\left[2 \arctan u_{n, x}(t, \xi(t, x))\right]=\frac{2}{1+u_{n, x}^{2}}\left[u_{n}^{2}-\frac{u_{n, x}^{2}}{2}-P^{u_{n}}\right] \leq-\frac{1}{2},
$$

whenever $u_{n, x}^{2}$ is sufficiently large. For $\varepsilon>0$ small, consider the piecewise affine, $2 \pi$-periodic function (see fig. 2 )

$$
\varphi(\theta)=\left\{\begin{array}{rll}
\theta & \text { if } & 0 \leq \theta \leq 1 \\
1 & \text { if } & 1 \leq \theta \leq \pi-\varepsilon \\
(\pi-\theta) / \varepsilon & \text { if } & \pi-\varepsilon \leq \theta \leq \pi+\varepsilon, \\
-1 & \text { if } & \pi+\varepsilon \leq \theta \leq 2 \pi-1, \\
\theta-2 \pi & \text { if } & 2 \pi-1 \leq \theta \leq 2 \pi .
\end{array}\right.
$$

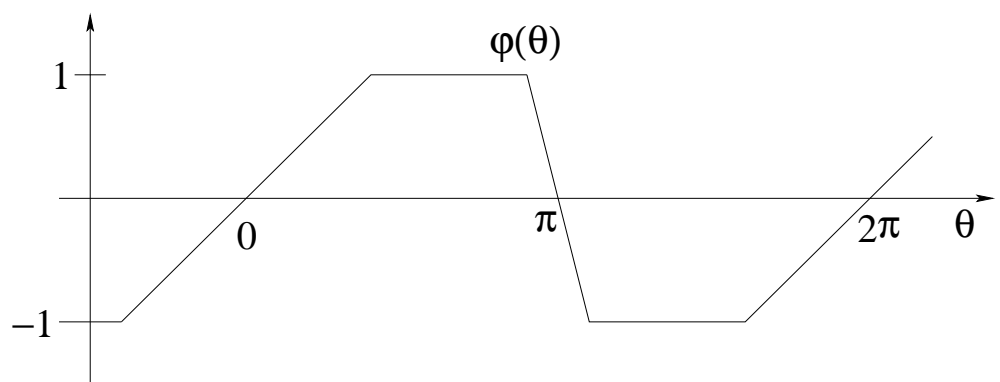

FiG. 2

and define

$$
\beta_{n}(t)=\int_{0}^{1} \varphi\left(2 \arctan u_{n, x}(t, x)\right) u_{n, x}^{2}(t, x) d x .
$$

By (1.3) and (6.6) we now have

$$
\begin{aligned}
\frac{d}{d t} \beta_{n}(t) \geq \frac{1}{4 \varepsilon} & \int_{\left\{2 \arctan u_{n, x} \in[\pi-\varepsilon, \pi] \cup[-\pi,-\pi+\varepsilon]\right\}} u_{n, x}^{2}(t, x) d x \\
& -C \cdot \int_{\left\{2 \arctan u_{n, x} \in[-1,1]\right\}} u_{n, x}^{2} d x
\end{aligned}
$$

for some constant $C$, independent of $\varepsilon, n$. Since all functions $\beta_{n}$ remain uniformly bounded, by $(6.7)$ for any time interval $\left[\tau, \tau^{\prime}\right]$ we obtain

$$
\int_{\tau}^{\tau^{\prime}} \int_{\left\{2 \arctan u_{n, x} \in[\pi-\varepsilon, \pi] \cup[-\pi,-\pi+\varepsilon]\right\}} u_{n, x}^{2}(t, x) d x d t \leq \varepsilon C^{\prime} \cdot\left(1+\tau^{\prime}-\tau\right),
$$


where the constant $C^{\prime}$ depends only on the $H_{\text {per }}^{1}$ norm of the functions $u_{n}$, hence is uniformly valid for all $n, \varepsilon$. Because of (6.8), the sequence of functions $u_{n, x}^{2}$ is equi-integrable on any domain of the form $\left[\tau, \tau^{\prime}\right] \times[0,1]$. Namely

$$
\lim _{\kappa \rightarrow \infty} \int_{\tau}^{\tau^{\prime}} \int_{\left\{x \in[0,1], u_{n, x}^{2}>\kappa\right\}} u_{n, x}^{2}(t, x) d x d t=0
$$

uniformly w.r.t. $n$. By Lemma 4 we already know that $\left\|u_{n, x}^{p}(t)-u_{x}^{p}(t)\right\|_{\mathbf{L}_{\text {per }}^{1}} \rightarrow 0$ for every fixed time $t$ and $1 \leq p<2$. Thanks to the equi-integrability condition (6.9) we now have

$$
u_{n, x}^{2} \rightarrow u_{x}^{2} \quad \text { in } \quad \mathbf{L}^{1}\left(\left[\tau, \tau^{\prime}\right] \times[0,1]\right) .
$$

By Fubini's theorem, this implies

$$
\lim _{n \rightarrow \infty} \int_{0}^{1} u_{n, x}^{2}(t, x) d x=\int_{0}^{1} u_{x}^{2}(t, x) d x
$$

for a.e. $t \in\left[\tau, \tau^{\prime}\right]$. At every such time $t$, the measure $\mu_{t}$ is absolutely continuous and the definition (6.3) coincides with (1.2). This completes the proof of Theorem 1.

7. Uniqueness. Before proving Theorem 3, we remark that the solution satisfying all conditions in Theorem 1 need not be unique.

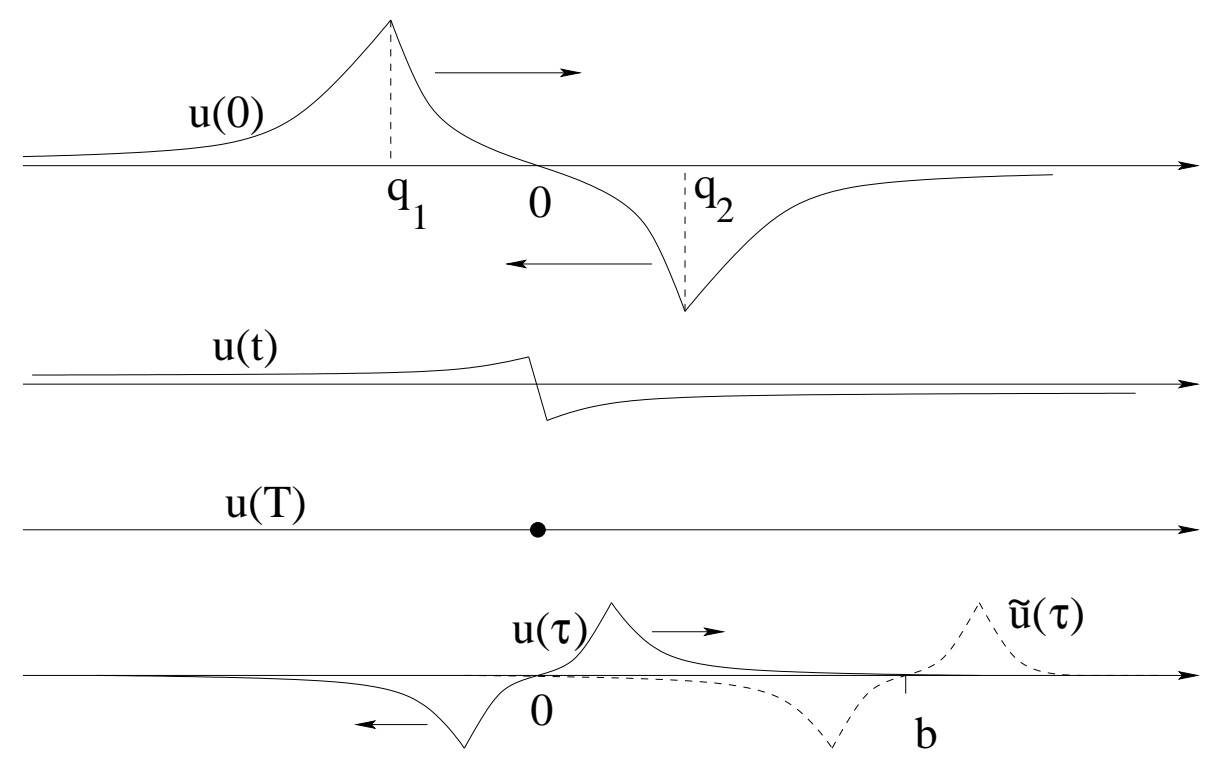

FIG. 3

EXAMPLE. Let $u=u(t, x)$ be a solution containing exactly two peakons of opposite strengths $p_{1}(t)=-p_{2}(t)$, located at points $q_{1}(t)=-q_{2}(t)$ (see fig. 3). We 
assume that initially $p_{1}(0)>0 q_{1}(0)<0$. At a finite time $T>0$, the two peakons interact at the origin. In particular, as $t \rightarrow T$ - there holds

$$
p_{1}(t) \rightarrow \infty, \quad p_{2}(t) \rightarrow-\infty, \quad q_{1}(t) \rightarrow 0, \quad q_{2}(t) \rightarrow 0 .
$$

Moreover, $\|u(t)\|_{\mathbf{L}_{\infty}} \rightarrow 0$, while the measure $\mu_{t}$ approaches a Dirac mass at the origin. We now have various ways to extend the solution beyond time $T$ :

$$
\begin{gathered}
\tilde{u}(\tau, x) \doteq 0, \\
u(\tau, x)=-u(\tau-T,-x),
\end{gathered}
$$

Clearly, $\tilde{u}$ dissipates all the energy, and does not satisfy the identity (1.15). The function $u$ in (7.2) is the one constructed by our algorithm in Section 2. However, there are infinitely many other solutions that still satisfy (1.15), for example

$$
\tilde{u}(\tau, x)=u(\tau, x-b)
$$

where $u$ is as in (7.2) and $b \neq 0$. The additional condition in Theorem 3 rules out all of them, because as $\tau \rightarrow T+$, the corresponding measures $\tilde{\mu}_{\tau}$ approach a Dirac mass at the point $x=b$, not at the origin.

We can now give a proof of Theorem 3. As a first step, we extend our distance $J$ to a larger domain $\mathcal{D}$, consisting of couples $(u, \mu)$, where $u \in H_{\text {per }}^{1}$ and $\mu$ is a positive (spatially periodic) measure whose absolutely continuous part has density $u^{2}+u_{x}^{2}$ w.r.t. Lebesgue measure. This extension is achieved by continuity:

$$
J((u, \mu),(\tilde{u}, \tilde{\mu})) \doteq \liminf _{n \rightarrow \infty} J\left(u_{n}, \tilde{u}_{n}\right)
$$

where the infimum is taken over all couple of sequences $\left(u_{n}, \tilde{u}_{n}\right)_{n \geq 1}$ such that

$$
\begin{array}{rlrl}
\left\|u_{n}-u\right\|_{\mathbf{L} \infty} & \rightarrow 0, & & \left\|\tilde{u}_{n}-\tilde{u}\right\|_{\mathbf{L} \infty} \rightarrow 0, \\
u_{n, x}^{2} \rightarrow \mu, & & \tilde{u}_{n, x}^{2} \rightarrow \tilde{\mu} .
\end{array}
$$

We observe that the flow $\Phi$ constructed in Theorem 2 can be continuously extended to a locally Lipschitz continuous group of transformations on the domain $\mathcal{D}$, so that

$$
J\left(\Phi_{t}(u, \mu), \Phi_{t}\left(u^{\prime}, \mu^{\prime}\right)\right) \leq e^{C_{2}|t|} J\left((u, \mu),\left(u^{\prime}, \mu^{\prime}\right)\right)
$$

Here one can choose a constant $C_{2}$ uniformly valid as $(u, \mu),\left(u^{\prime}, \mu^{\prime}\right)$ range over bounded subsets of $\mathcal{D}$. Now let $t \mapsto u(t)$ be a solution of the Cauchy problem (1.1), (1.13), satisfying all the required conditions. In particular, the map $t \mapsto\left(u(t), \mu_{t}\right)$ is Lipschitz continuous w.r.t. the distance $J$, with values in the domain $\mathcal{D}$. Calling $t \mapsto\left(\tilde{u}(t), \tilde{\mu}_{t}\right) \doteq$ $\Phi_{t}\left(\bar{u}, \bar{u}_{x}^{2}\right)$ the unique solution of the Cauchy problem obtained as limit of multi-peakon approximations, we need to show that $u(t)=\tilde{u}(t)$ and $\mu_{t}=\tilde{\mu}_{t}$ for all $t$. To fix the ideas, let $t>0$. By the Lipschitz continuity of the flow, stated at (7.5), we can use the error estimate

$$
J\left(\left(u(t), \mu_{t}\right),\left(\tilde{u}(t), \tilde{\mu}_{t}\right)\right) \leq e^{C_{2} t} \int_{0}^{t} \liminf _{h \rightarrow 0} \frac{J\left(\left(u(\tau+h), \mu_{\tau+h}\right), \Phi_{h}\left(u(\tau), \mu_{\tau}\right)\right)}{h} d \tau .
$$


For a proof of (7.6), see pp. $25-27$ in [B]. The conditions stated in Theorem 1 now imply that, at almost every time $\tau$, the measure $\tilde{\mu}_{t}$ is absolutely continuous and the integrand in (7.5) vanishes. Therefore $\tilde{u}(t)=u(t)$ for all $t$.

We can now prove that, in multi-peakon solutions, interactions involving exactly two peakons are the only possible ones.

Corollary. Let $t \mapsto u(t, j$ be a multi-peakon solution of the form (1.7), which remains regular on the open interval $] 0, T[$. Assume that at time $T>0$ an interaction occurs, say among the first $k$ peakons, so that

$$
\lim _{t \rightarrow T-} q_{i}(t)=\bar{q} \quad i=1, \ldots, k .
$$

Then $k=2$.

Proof. We first observe that the Camassa-Holm equations (1.1) are time-reversible. In particular, our proof of Theorem 3 shows that the solution to a Cauchy problem is unique both forward and backward in time.

Now consider the data $\left(u(T), \mu_{T}\right) \in \mathcal{D}$, where $\mu_{T}$ is the weak limit of the measures $\mu_{t}$ having density $u^{2}(t)+u_{x}^{2}(t)$ w.r.t. Lebesgue measure, as $t \rightarrow T-$. By the analysis in Section 2, we can construct a backward solution of this Cauchy problem in terms of exactly two incoming peakons. By uniqueness, this must coincide with the given solution $u(\cdot)$ for all $t \in[0, T]$.

\section{REFERENCES}

[B] A. Bressan, Hyperbolic Systems of Conservation Laws. The One Dimensional Cauchy Problem, Oxford University Press, 2000.

[BC1] A. Bressan and A. Constantin, Global solutions to the Hunter-Saxton equations, SIAM J. Math. Anal., 37 (2005), pp. 996-1026.

[BC2] A. Bressan And A. Constantin, Global solutions of the Camassa-Holm equations, Archive Rat. Mech. Anal., to appear.

[BZZ] A. Bressan, P. Zhang, And Y. Zheng, On asymptotic variational wave equations, Arch. Rat. Mech. Anal., to appear.

[CHK1] G. M. Coclite, H. Holden And K. H. Karlsen, Well-posedness for a parabolic-elliptic system, Discrete Contin. Dynam. Systems., 13 (2005), pp. 659-682.

[CHK2] G. M. Coclite, H. Holden And K. H. KARLSEn, Global weak solutions to a generalized hyperelastic-rod wave equation, submitted.

[CH] R. Camassa And D. D. Holm, An integrable shallow water equation with peaked solitons, Phys. Rev. Lett., 71 (1993), pp. 1661-1664.

[C1] A. Constantin, Existence of permanent and breaking waves for a shallow water equation: a geometric approach, Ann. Inst. Fourier (Grenoble), 50 (2000), pp. 321-362.

[C2] A. Constantin, On the scattering problem for the Camassa-Holm equation, Proc. Roy. Soc. London Ser. A, 457 (2001), pp. 953-970.

[CE1] A. Constantin And J. Escher, Global existence and blow-up for a shallow water equation, Ann. Scuola Norm. Sup. Pisa, 26 (1998), pp. 303-328.

[CE2] A. CONSTANTin AND J. EscheR, Wave breaking for nonlinear nonlocal shallow water equations, Acta Mathematica, 181 (1998), pp. 229-243.

[CM1] A. Constantin And H. P. McKean, A shallow water equation on the circle, Comm. Pure Appl. Math., 52 (1999), pp. 949-982.

[CM2] A. Constantin And L. Molinet, Global weak solutions for a shallow water equation, Comm. Math. Phys., 211 (2000), pp. 45-61.

[EG] L. C. Evans and R. F. Gariepy, Measure Theory and Fine Properties of Functions, Studies in Advanced Mathematics, CRC Press, Boca Raton, FL, 1992. 
[J] R. S. Johnson, Camassa-Holm, Korteweg-de Vries and related models for water waves, J. Fluid Mech., 455 (2002), pp. 63-82.

[V] C. Villani, Topics in Optimal Transportation, Amer. Math. Soc., Providence 2003.

[XZ] Z. XIN AND P. ZHANG, On the weak solutions to a shallow water equation, Comm. Pure Appl. Math., 53 (2000), pp. 1411-1433.

[XZ2] Z. XIN AND P. ZHANG, On the uniqueness and large time behavior of the weak solutions to a shallow water equation, Comm. Partial Differential Equations, 27 (2002), pp. 18151844 . 
